\title{
Gravitational Radiation Reaction to a Particle Motion
}

\author{
Yasushi Mino, ${ }^{1,2}$ 田 Misao Sasaki, ${ }^{1}$ ] and Takahiro Tanaka ${ }^{1}$. \\ ${ }^{1}$ Department of Earth and Space Science, Graduate School of Science \\ Osaka University, Toyonaka 560, Japan \\ ${ }^{2}$ Department of Physics, Faculty of Science, Kyoto University, Kyoto 606-01, Japan
}

\begin{abstract}
A small mass particle traveling in a curved spacetime is known to trace a background geodesic in the lowest order approximation with respect to the particle mass. In this paper, we discuss the leading order correction to the equation of motion of the particle, which presumably describes the effect of gravitational radiation reaction.

We derive the equation of motion in two different ways. The first one is an extension of the well-known formalism by DeWitt and Brehme developed for deriving the equation of motion of an electrically charged particle. Constructing the conserved rank two symmetric tensor, and integrating it over the interior of the world tube surrounding the orbit, we derive the equation of motion. Although the calculation in this approach is straightforward, it contains less rigorous points. In contrast to the electromagnetic case, in which there are two different charges, i.e., the electric charge and the mass, the gravitational counterpart has only one charge. This fact prevents us from using the same renormalization scheme that was used in the electromagnetic case. In order to overcome this difficulty, we put an ansatz in evaluating the integral of the conserved tensor on a three spatial volume which defines the momentum of the small particle.

To make clear the subtlety in the first approach, we then consider the asymptotic matching of two different schemes, i.e., the internal scheme in which the small particle is represented by a spherically symmetric black hole with tidal perturbations and the external scheme in which the metric is given by small perturbations on the given background geometry. The equation of motion is obtained from the consistency condition of the matching. We find that in both ways the same equation of motion is obtained. The resulting equation of motion is analogous to that derived in the electromagnetic case. We discuss implications of this equation of motion.
\end{abstract}

PACS number(s): 04.30.Db, 04.25.-g

\section{INTRODUCTION}

The gravitational waves from an inspiralling binary is one of the most promising sources expected to be detected by the near-future interferometric gravitational wave detectors such as LIGO, VIRGO and LISA [1.22]. In order to extract the information of the binaries from the last inspiralling stage, we need accurate theoretical templates of the gravitational wave forms [3]. As an approach, perturbations of a black hole by an orbiting small particle has been studied 沺. In all the previous works, the rate of change of orbital parameters are assumed to be determined by the energy balance. Although this prescription is powerful, the background black hole is required to have a sufficient number of Killing vector fields in order to relate the outgoing gravitational waves with the rate of change of orbital parameters of the particle. The Kerr black hole is an important example which does not have a sufficient number of

\footnotetext{
*Electronic address: mino@vega.ess.sci.osaka-u.ac.jp

${ }^{\dagger}$ Electronic address: misao@vega.ess.sci.osaka-u.ac.jp

${ }^{\ddagger}$ Electronic address: tama@vega.ess.sci.osaka-u.ac.jp
} 
Killing vector fields and so rigorous discussions are restricted to the case in which the particle is in a circular or a equatorial orbit. Thus the effect of the radiation reaction on the motion of a small mass particle in a general curved spacetime is an important target of theoretical investigation.

On the other hand, the effect of the electromagnetic radiation reaction on the motion of a charged particle in a curved spacetime was discussed by DeWitt and Brehme [5] (Hereafter DB). In the electromagnetic case, the total energy momentum tensor composed of the particle and field contributions satisfies the conservation law. Its divergence is integrated over the interior of the tube surrounding the particle orbit with infinitesimal length. The part of the integration which does not vanish in the small tube radius limit is transformed into the surface integrations over the both ends of the tube and over the surface of the tube by using the Gauss' theorem. The integrations over the top and bottom of the tube give the definition of the particle momenta at both ends and the difference between them represents the change of the momentum during this infinitesimal time interval, which is to be equated with the momentum flow given by the integration over the surface of the tube. In this way the equation of motion is obtained. As shown below, also in the case of gravitational radiation reactions, we can construct a conserved rank two tensor defined on the background spacetime, composed of the matter field and the metric perturbation. However, there is an important difference between electromagnetic and gravitational cases. In the electromagnetism, we can consider an extended charge distribution which is supported by a certain force other than the electromagnetic field. Thus it will be natural to assume that the charge and mass distributions of a point-like particle are not distorted by the effect of the radiation reaction. Therefore one may consistently assume that the momentum and the electric current of the particle are proportional to the 4-velocity of the particle. Moreover the electromagnetic charge $e$ is not directly related to the energy momentum of the particle which is proportional to the mass $m$. Hence, even if the limit of zero particle radius is taken, the divergent self-energy, $\left(\propto e^{2}\right)$ can be renormalized by shifting the bare mass. In the case of the gravitational radiation reaction, it is not possible to consider such an ideal point-like particle because every force field universally couples with gravity. Even worse, the role of $e$ in the electromagnetism is also attributed to $m$. Thus the simple renormalization scheme does not make any sense. In order to overcome this difficulty, we put an ansatz that the particle momentum, defined in a similar way as in the electromagnetism but without taking the limit of the small particle radius, be proportional to the 4-velocity of the particle, which will not be justified within this framework. Under this assumption, we obtain an equation of motion in the covariant form with respect to the background spacetime which is analogous to that obtained in the case of electromagnetic case.

In order to develop a more rigorous formalism, we consider an extension of the matched asymptotic expansion that has been already developed by many authors (e.g., D'Eath [6] and Thorne and Hartle [7]). We assume that the internal metric which describes the geometry around the particle is represented by a Schwarzschild black hole of small mass in the lowest order approximation. As the particle moves in the curved background, it suffers from the tidal distortion. This effect is taken into account by the homogeneous linear perturbations of the black hole. Since we know $\ell=0$ and 1 homogeneous perturbations of the black hole are purely indebted to gauge degrees of freedom as long as both the mass and angular momentum of the black hole stay constant, we set them to vanish. We also assume that the external metric is approximated by the linear perturbations on the background spacetime generated by a point-like particle. Then we consider a limited class of coordinate transformation which keeps the meaning of the center of the particle unambiguous and match the external metric with the internal one in the matching region where the both approximations are valid. Then we find that for an arbitrary orbit the consistent coordinate transformation does not exist, and this consistency condition determines the equation of motion, which is no different from that obtained in the first approach.

This paper is organized as follows. In section 2 we explain the covariant expansion method of the tensor Green function, which becomes important in both approaches discussed in the succeeding two sections. In section 3 we discuss the first approach, i.e., an extension of DeWitt and Brehme's electromagnetic radiation reaction equation of motion to the gravitational counter part. Section 4 is devoted to the second approach, i.e., the matched asymptotic expansion method to derive the equation of motion of a small black hole. We find both approaches give the same equation of motion. In section 5 we discuss implications of the result. Section 6 is devoted to conclusion.

We suppose the reader is familiar with the concept of 'bi-tensors' and some useful tools developed by DB. Following $\mathrm{DB}$, we assign the indices $\alpha, \beta, \gamma, \delta, \epsilon, \zeta, \eta$ for the point on the particle trajectory, $z(\tau)$, and the indices $\mu, \nu, \xi, \rho, \sigma$ for the field point, $x$. For the reader's convenience, the notation and basic formulas are summarized in Appendix A.

\section{METRIC PERTURBATION}

In this section, for the later use, we calculate the metric perturbation, $\delta g_{\mu \nu}$, induced by a point-like particle on the background metric, $g_{\mu \nu}$. The background metric is assumed to satisfy the vacuum Einstein equations. Thus, in the following calculations, we use the fact that the background Ricci tensor vanishes. As we assume that the particle mass, $m$, is small compared with the background curvature scale, $L$, we approximate $\delta g_{\mu \nu}$ by the linear perturbation induced by a point-like particle, $h_{\mu \nu}$, in the whole spacetime region except for the vicinity of the world line of the particle. We call the region in which this approximation is valid as the external zone. On the other hand, in the vicinity of the world line of the particle, the self gravity of the particle dominates and the metric cannot be described by the linear perturbation induced by a point-like particle. We call this region as the internal zone. In this section we concentrate on the external zone. The calculation is performed in an analogous manner to the case of the scalar and vector perturbations developed by DB.

Here we consider the linearized Einstein equations. We introduce the trace-reversed metric perturbation, 


$$
\psi_{\mu \nu}(x)=h_{\mu \nu}(x)-\frac{1}{2} g_{\mu \nu}(x) h(x)
$$

and set the harmonic gauge condition,

$$
\psi_{; \nu}^{\mu \nu}(x)=0,
$$

where $h(x)$ and $\psi(x)$ are the trace of $h^{\mu \nu}(x)$ and that of $\psi^{\mu \nu}(x)$, respectively, and the semicolon means the covariant derivative with respect to the background metric. In this gauge, the linearized Einstein equations become

$$
-\frac{1}{2} \psi^{\mu \nu ; \xi} \xi(x)-R_{\xi}^{\mu}{ }_{\rho}^{\nu}(x) \psi^{\xi \rho}(x)=8 \pi G T^{\mu \nu}(x)+O\left(\mathbf{h}^{2}\right),
$$

where $O\left(\mathbf{h}^{2}\right)$ stands for terms quadratic or of higher powers in the metric perturbation. Thus we define the tensor Green function $G^{\mu \nu \alpha \beta}(x, z)$ which satisfies

$$
G^{\mu \nu \alpha \beta ; \xi} ; \xi(x, z)+2 R^{\mu}{ }_{\xi}^{\nu}{ }_{\rho}(x) G^{\xi \rho \alpha \beta}(x, z)=-2 \bar{g}^{\alpha(\mu}(x, z) \bar{g}^{\nu) \beta}(x, z) \frac{\delta^{(4)}(z-x)}{\sqrt{-g}},
$$

where $\bar{g}^{\mu \alpha}(x, z)$ is the bi-vector of geodetic parallel displacement defined in Eq. (A7) of Appendix A and $g$ is the determinant of the metric $g_{\mu \nu}(x)$.

First we consider the elementary solution $G_{*}^{\mu \nu \alpha \beta}(x, z)$ which satisfies Eq. (2.4) except at the $\sigma(x, z) \rightarrow 0$ limit and takes the Hadamard form,

$$
G_{*}^{\mu \nu \alpha \beta}(x, z)=\frac{1}{(2 \pi)^{2}}\left(\frac{u^{\mu \nu \alpha \beta}(x, z)}{\sigma(x, z)}+v^{\mu \nu \alpha \beta}(x, z) \log |\sigma(x, z)|+w^{\mu \nu \alpha \beta}(x, z)\right),
$$

where $\sigma(x, z)$ is a half of the square of geodetic interval which is defined in Eq. (1.10) of DB. Its property is summarized in Eq. (A6) of Appendix A. The functions $u^{\mu \nu \alpha \beta}(x, z), v^{\mu \nu \alpha \beta}(x, z)$ and $w^{\mu \nu \alpha \beta}(x, z)$ are bi-tensors which are regular in the $\sigma(x, z) \rightarrow 0$ limit and $u^{\mu \nu \alpha \beta}(x, z)$ satisfies the normalization condition,

$$
\lim _{x \rightarrow z} u^{\mu \nu \alpha \beta}(x, z)=\lim _{x \rightarrow z} 2 \bar{g}^{\alpha(\mu}(x, z) \bar{g}^{\nu) \beta}(x, z) .
$$

If we put the form (2.5) into the left hand side of Eq. (2.4), the terms can be classified into three parts. One is the terms which contain the factor $1 / \sigma^{2}(x, z)$ manifestly and another is the terms which contain $\log |\sigma(x, z)|$. The remaining terms have no singular behavior at the $\sigma(x, z) \rightarrow 0$ limit. Since the form (2.5) is redundant, we can set these three sets to vanish separately:

$$
\begin{aligned}
& \left(2 u^{\mu \nu \alpha \beta ; \xi}(x, z)-\frac{\Delta^{; \xi}(x, z)}{\Delta(x, z)} u^{\mu \nu \alpha \beta}(x, z)\right) \sigma_{; \xi}(x, z)=0, \\
& v^{\mu \nu \alpha \beta ; \xi} ; \xi(x, z)+2 R^{\mu} \xi_{\rho}^{\nu}(x) v^{\xi \rho \alpha \beta}(x, z)=0, \\
& 2 v^{\mu \nu \alpha \beta}(x, z)+\left(2 v^{\mu \nu \alpha \beta ; \xi}(x, z)-\frac{\Delta^{; \xi}(x, z)}{\Delta(x, z)} v^{\mu \nu \alpha \beta}(x, z)\right) \sigma_{; \xi}(x, z)+u^{\mu \nu \alpha \beta ; \xi} ; \xi(x, z) \\
& \quad+2 R_{\xi}^{\mu}{ }_{\rho}^{\nu}(x) u^{\xi \rho \alpha \beta}(x, z)+\left(w^{\mu \nu \alpha \beta ; \xi}{ }_{\xi}(x, z)+2 R_{\xi}^{\mu}{ }_{\xi}^{\nu}(x) w^{\xi \rho \alpha \beta}(x, z)\right) \sigma(x, z)=0,
\end{aligned}
$$

where we used the bi-scalar $\Delta(x, z)$ defined in Eq. (A8) in Appendix A. Equation (2.7) is solved with the normalization (2.6) as

$$
u^{\mu \nu \alpha \beta}(x, z)=2 \bar{g}^{\alpha(\mu}(x, z) \bar{g}^{\nu) \beta}(x, z) \sqrt{\Delta(x, z)} .
$$

The functions $v^{\mu \nu \alpha \beta}(x, z)$ and $w^{\mu \nu \alpha \beta}(x, z)$ are to be determined by solving Eqs. (2.8) and (2.9). The function $w^{\mu \nu \alpha \beta}(x, z)$ is not needed but the function $v^{\mu \nu \alpha \beta}(x, z)$ plays an important role in the following discussion. Although it is difficult to find the solution of $v^{\mu \nu \alpha \beta}(x, z)$ in an arbitrary background spacetime, its explicit form is not required for the succeeding discussions. However it is important to note that $v^{\mu \nu \alpha \beta}(x, z)$ is uniquely determined. The reason is as follows. From Eq. (2.8) one finds it satisfies a hyperbolic equation. Hence the problem is if its Cauchy data are unique or not. First we note the coincidence limit of Eq. (2.9), which gives

$$
\lim _{x \rightarrow z} v^{\mu \nu \alpha \beta}(x, z)=-\lim _{x \rightarrow z} 2 \bar{g}_{(\xi}^{\alpha}(z, x) \bar{g}_{\rho)}^{\beta}(z, x) R^{\mu \xi \nu \rho}(x) .
$$


Then taking the null limit $\sigma(x, z) \rightarrow 0$ of Eq. (2.9), we obtain the first order differential equation of $v^{\mu \nu \alpha \beta}(x, z)$ which can be solved along the null geodesic. Thus this equation with the boundary condition (2.11) uniquely determines $v^{\mu \nu \alpha \beta}(x, z)$ on the light cone emanating from $z$. Therefore the hyperbolic equation (2.8) has a unique solution. We also mention that $v^{\mu \nu \alpha \beta}(x, z)$ is divergence free,

$$
v_{; \nu}^{\mu \nu \alpha \beta}(x, z)=0 .
$$

To see this we note the harmonic gauge condition on the Green function requires

$$
\lim _{\sigma \rightarrow 0} v_{; \nu}^{\mu \nu \alpha \beta}(x, z)=0 .
$$

We also see that the equation for $v_{; \nu}^{\mu \nu \beta}(x, z)$ follows from Eq. (2.8),

$$
\left[v_{; \nu}^{\mu \nu \alpha \beta}(x, z)\right]_{; \xi}^{; \xi}=0
$$

where we have used the fact $R^{\mu \xi \nu \rho} ; \rho=0$, which is proved by contracting the Bianchi identities for the vacuum case. Thus we conclude that Eq. (2.12) holds everywhere.

The Feynman propagator $G_{F}^{\mu \nu \alpha \beta}(x, z)$ can be derived from the elementary solution $G_{*}^{\mu \nu \alpha \beta}(x, z)$ by the $i \epsilon$ prescription.

$$
G_{F}^{\mu \nu \alpha \beta}(x, z)=\frac{1}{(2 \pi)^{2}}\left(\frac{u^{\mu \nu \alpha \beta}(x, z)}{\sigma(x, z)+i \epsilon}+v^{\mu \nu \alpha \beta}(x, z) \log (\sigma(x, z)+i \epsilon)+w^{\mu \nu \alpha \beta}(x, z)\right) .
$$

The imaginary part of the Feynman propagator $G_{F}^{\mu \nu \alpha \beta}(x, z)$ gives the symmetric Green function $\bar{G}^{\mu \nu \alpha \beta}(x, z)$, from which we can obtain the retarded Green function $G_{R e t}^{\mu \nu \alpha \beta}(x, z)$, and the advanced Green function $G_{A d v}^{\mu \nu \alpha \beta}(x, z)$ as

$$
\begin{aligned}
\bar{G}^{\mu \nu \alpha \beta}(x, z) & =-\frac{1}{2} \operatorname{Im}\left[G_{F}^{\mu \nu \alpha \beta}(x, z)\right] \\
& =\frac{1}{8 \pi}\left[u^{\mu \nu \alpha \beta}(x, z) \delta(\sigma(x, z))-v^{\mu \nu \alpha \beta}(x, z) \theta(-\sigma(x, z))\right], \\
G_{R e t}^{\mu \nu \alpha \beta}(x, z) & =2 \theta[\Sigma(x), z] \bar{G}^{\mu \nu \alpha \beta}(x, z), \\
G_{A d v}^{\mu \nu \alpha \beta}(x, z) & =2 \theta[z, \Sigma(x)] \bar{G}^{\mu \nu \alpha \beta}(x, z),
\end{aligned}
$$

where $\Sigma(x)$ is an arbitrary space-like hypersurface containing $x$, and $\theta[\Sigma(x), z]=1-\theta[z, \Sigma(x)]$ is equal to 1 when $z$ lies in the past of $\Sigma(x)$ and vanishes when $z$ lies in the future.

Now, using the above obtained Green functions, we compute the trace-reversed metric perturbation $\psi^{\mu \nu}(x)$ induced by a point-like particle whose energy momentum tensor is given by

$$
T^{\mu \nu}(x)=m \int d \tau v^{\mu}(x, \tau) v^{\nu}(x, \tau) \frac{\delta^{(4)}(x-z(\tau))}{\sqrt{-g}}
$$

where $v^{\mu}(x, \tau)=\bar{g}^{\mu}{ }_{\alpha}(x, z(\tau)) \dot{z}^{\alpha}(\tau)$ and $\dot{z}^{\alpha}(\tau)=d z^{\alpha} / d \tau$. It is also assumed that the particle mass $m$ is small compared with the background curvature scale $L$. Throughout this paper, we take the unit in which $L$ becomes of $O(1)$. There appear relations whose dimensionality looks wrong but in those cases $L$ is omitted for notational simplicity.

At this point, we must comment on the reason why we consider the point-like particle. Even in the linear perturbation, in order to generate a general gravitational field in the external zone, we need to consider a source with arbitrary higher multipole moments. However, in the following discussion, we are going to discuss the situation in which those higher moments are negligible. Thus we consider this special case here. Later it will become clarified in what situation this assumption becomes consistent.

Now, for the point-like particle, the trace-reversed metric perturbation $\psi^{\mu \nu}(x)$ with the retarded or advanced boundary condition is computed as

$$
\begin{aligned}
\psi_{\text {Ret } / A d v}^{\mu \nu}(x)= \pm 2 G m( & {\left[\frac{1}{\dot{\sigma}(x, z(\tau))} u_{\alpha \beta}^{\mu \nu}(x, z(\tau)) \dot{z}^{\alpha}(\tau) \dot{z}^{\beta}(\tau)\right]_{\tau=\tau_{\text {Ret } / A d v}(x)} } \\
& \left.-\int_{\mp \infty}^{\tau_{\text {Ret/Adv }}(x)} d \tau v^{\mu \nu}{ }_{\alpha \beta}(x, z(\tau)) \dot{z}^{\alpha}(\tau) \dot{z}^{\beta}(\tau)\right) .
\end{aligned}
$$

where $\tau_{\operatorname{Ret} / A d v}(x)$ is the retarded or advanced time of the particle and is a scalar function which is determined by 


$$
\begin{aligned}
& \sigma\left(x, z\left(\tau_{\text {Ret }} / A d v\right)\right)=0 \\
& \theta\left(\Sigma(x), z\left(\tau_{\text {Ret }}\right)\right)=\theta\left(z\left(\tau_{A d v}\right), \Sigma(x)\right)=1 .
\end{aligned}
$$

Throughout this paper, we take the convention that the upper sign is assigned for the retarded boundary condition and the lower sign is for the advanced one.

Since the expression (2.20) containing the retarded or advanced time $\tau_{\operatorname{Ret} / A d v}(x)$ is not convenient for the computations in the succeeding sections, we rewrite it by introducing a new parameterization of the field point $x$. We foliate the spacetime with spacelike 3-surfaces perpendicular to the particle trajectory. More strictly, the 3-surfaces are defined as a one-parameter family of $\tau$ by the relation, $\sigma_{; \alpha}(x, z(\tau)) \dot{z}^{\alpha}(\tau)=0$. We denote the value of $\tau$ of the 3 -surface containing the point $x$ by $\tau_{x}$. That is

$$
\left[\sigma_{; \alpha}(x, z(\tau)) \dot{z}^{\alpha}(\tau)\right]_{\tau=\tau_{x}}=0
$$

To distinguish the spatial points on the same 3-surface, we use $\sigma_{; \alpha}\left(x, z\left(\tau_{x}\right)\right)$ and denote the distance between $x$ and $z\left(\tau_{x}\right)$ by

$$
\epsilon(x):=\sqrt{2 \sigma\left(x, z\left(\tau_{x}\right)\right)}
$$

As we are interested in the region where the linear perturbation is valid, $\epsilon(x)$ must be much greater than $G m$. However, concentrating on the region close to the particle even in the external zone, we can take $\epsilon(x)$ is smaller than the background curvature scale $L$, because $G m$ is assumed to be much smaller than $L$. Thus we can consider the expansion assuming that $\epsilon(x)$ is small.

In the following calculation there appear the higher derivatives of $\dot{z}$, such as $\ddot{z}$ and $\dddot{z}$, where a dot means the covariant derivative along the trajectory of the particle. Since we are considering the case in which the deviation of particle trajectory from the geodesic is a small correction due to the radiation reaction, we suppose that those higher derivatives are at most of $O(\epsilon(x))$. Thus we define the inverse of the reaction time scale $\tau_{r}^{-1}(<\epsilon(x))$ as the scale of $\ddot{z}, \dddot{z}$ and so on. We will see later that $\tau_{r}^{-1} \approx G m$ and this assumption is found to be consistent.

We first consider the time retardation or advancement, $\delta_{\text {Ret } / A d v}(x)$,

$$
\delta_{\text {Ret } / A d v}(x):=\tau_{\text {Ret } / A d v}(x)-\tau_{x} .
$$

The time retardation or advancement, $\delta_{\text {Ret } / A d v}(x)$, is expanded with respect to $\epsilon(x)$,

$$
\begin{gathered}
\delta_{\text {Ret } / A d v}(x)=\mp \epsilon(x) \kappa^{-1}(x)\left(1 \mp \frac{1}{6} \epsilon(x) \kappa^{-3}(x) \dddot{z}^{\alpha}\left(\tau_{x}\right) \sigma_{; \alpha}\left(x, z\left(\tau_{x}\right)\right)\right. \\
\left.-\frac{1}{24} \epsilon^{2}(x) \kappa^{-4}(x) \ddot{z}^{2}\left(\tau_{x}\right)\right)+O\left(\epsilon^{4}\right),
\end{gathered}
$$

as given in Eq. (4.40) of DB [3, where $\kappa^{2}(x)=-[\ddot{\sigma}(x, z(\tau))]_{\tau=\tau_{x}}$. Then the expression for the trace-reversed metric perturbation (2.20) becomes

$$
\begin{aligned}
& \psi_{\mu \nu}(x)= \pm 2 G m \bar{g}_{\mu \alpha} \bar{g}_{\nu \beta} \\
& \left( \pm \frac{2}{\epsilon} \kappa^{-1} \dot{z}^{\alpha} \dot{z}^{\beta}-4 \dot{z}^{(\alpha} \ddot{z}^{\beta)}-2 \dot{z}^{\gamma} \sigma^{; \delta} \dot{z}^{\epsilon} R_{\gamma \delta \epsilon}{ }^{(\alpha} \dot{z}^{\beta)} \mp 2 \epsilon R_{\gamma}^{\alpha \beta} \dot{z}^{\gamma} \dot{z}^{\delta}\right. \\
& -\int_{\mp \infty}^{\tau_{x}} d \tau^{\prime} v^{\alpha \beta} \alpha^{\prime} \beta^{\prime}\left(z\left(\tau_{x}\right), z\left(\tau^{\prime}\right)\right) \dot{z}^{\alpha^{\prime}}\left(\tau^{\prime}\right) \dot{z}^{\beta^{\prime}}\left(\tau^{\prime}\right) \\
& \left.+\sigma_{; \gamma} \int_{\mp \infty}^{\tau_{x}} d \tau^{\prime} v_{\alpha^{\prime} \beta^{\prime}}^{\alpha \beta}\left(z\left(\tau_{x}\right), z\left(\tau^{\prime}\right)\right) \dot{z}^{\alpha^{\prime}}\left(\tau^{\prime}\right) \dot{z}^{\beta^{\prime}}\left(\tau^{\prime}\right)\right)+O\left(\epsilon^{2}, \tau_{r}^{-1} \epsilon\right) .
\end{aligned}
$$

In the above and in what follows, we omit the suffix Ret/Adv to $\psi_{\mu \nu}$ for notational simplicity. The detailed derivation of the above formula is provided in Appendix A. Hereafter we also suppress the arguments $x, z\left(\tau_{x}\right)$ and $\tau_{x}$ unless there arises ambiguity.

The covariant derivative of the metric perturbation becomes also necessary in section 3 . For this purpose, we calculate the covariant derivatives of the quantities appearing in Eq. (2.27) such as $\tau_{x}, \epsilon(x)$ and so on. With the aid

${ }^{\S} \mathrm{In} \mathrm{DB}, \sigma_{; \alpha}\left(x, z\left(\tau_{x}\right)\right)$ is replaced by $-\epsilon(x) n_{i \alpha}\left(\tau_{x}\right) \Omega_{i}$. 
of the relations given in Appendix A, the derivative of $\tau_{x}$ can be calculated by taking the derivative of the both sides of the identity (2.23) as

$$
\tau_{x ; \mu}=\kappa^{-2} \bar{g}_{\mu \alpha}\left(-\dot{z}^{\alpha}+\frac{1}{6} R_{\beta \gamma \delta}^{\alpha} \sigma^{; \beta} \dot{z}^{\gamma} \sigma^{; \delta}\right)+O\left(\epsilon^{3}\right) .
$$

One must be careful that $\left[f\left(x, z\left(\tau_{x}\right)\right)\right]_{; \mu} \neq\left[f(x, z(\tau))_{; \mu}\right]_{\tau=\tau_{x}}$ since $\tau_{x}$ is $x$ dependent through Eq. (2.23). By using Eq. (2.28) and the relations given in Appendix A, we obtain

$$
\begin{aligned}
\epsilon_{; \mu}(x)= & -\frac{1}{\epsilon} \bar{g}_{\mu \alpha} \sigma^{; \alpha} \\
{\left[\sigma^{; \alpha}\left(z\left(\tau_{x}\right), x\right)\right]_{; \mu}=} & \bar{g}_{\mu \beta}\left(-g^{\alpha \beta}-\kappa^{-2} \dot{z}^{\alpha} \dot{z}^{\beta}\right. \\
& \left.+\frac{1}{6}\left(g^{\alpha \gamma} g^{\beta \epsilon}-2 g^{\alpha \gamma} \dot{z}^{\beta} \dot{z}^{\epsilon}+\dot{z}^{\alpha} g^{\beta \gamma} \dot{z}^{\epsilon}\right) R_{\gamma \delta \epsilon \zeta} \sigma^{; \delta} \sigma^{; \zeta}\right)+O\left(\epsilon^{2}\right), \\
{\left[\bar{g}^{\mu \alpha}\left(x, z\left(\tau_{x}\right)\right)\right]_{; \nu}=} & \frac{1}{2} \bar{g}_{\beta}^{\mu} \bar{g}_{\nu \gamma}\left(g^{\gamma \delta}-\dot{z}^{\gamma} \dot{z}^{\delta}\right) R^{\alpha \beta}{ }_{\delta \epsilon} \sigma^{; \epsilon}+O\left(\epsilon^{2}\right), \\
\kappa_{; \nu}(x)= & \frac{1}{2 \kappa}\left[-\ddot{\sigma}\left(x, z\left(\tau_{x}\right)\right)\right]_{; \nu} \\
= & \frac{1}{2 \kappa} \bar{g}_{\nu \alpha}\left(\ddot{z}^{\alpha}+\dot{z}^{\alpha} \sigma_{; \beta} \dddot{z} \beta+\frac{2}{3} R_{\beta \gamma \delta}^{\alpha} \dot{z}^{\beta} \sigma^{; \gamma} \dot{z}^{\delta}\right)+O\left(\epsilon^{3}\right) .
\end{aligned}
$$

Then using these results and Eq. (2.11), after some computations, we obtain the 1st and 2nd derivatives of the metric perturbation $\psi_{\mu \nu}(x)$.

$$
\begin{aligned}
& \psi_{\mu \nu ; \xi}(x)=2 G m \bar{g}_{\mu \alpha} \bar{g}_{\nu \beta} \bar{g}_{\xi \gamma} \\
& \left(\frac{2}{\epsilon^{3}} \kappa^{-1} \dot{z}^{\alpha} \dot{z}^{\beta} \sigma^{; \gamma}-\frac{1}{\epsilon} \dot{z}^{\alpha} \dot{z}^{\beta} \ddot{z}^{\gamma}-\frac{4}{\epsilon} \dot{z}^{(\alpha} \ddot{z}^{\beta)} \dot{z}^{\gamma} \pm 2 \dot{z}^{(\alpha} R^{\beta)}{ }_{\delta}^{\gamma}{ }_{\epsilon} \dot{z}^{\delta} \dot{z}^{\epsilon} \mp 2 R^{\alpha}{ }_{\delta}{ }^{\beta} \dot{z}^{\gamma} \dot{z}^{\delta} \dot{z}^{\epsilon}\right. \\
& -\frac{2}{\epsilon} \dot{z}^{(\alpha} R^{\beta)}{ }_{\delta}{ }^{\gamma} \dot{\epsilon}^{\delta} \sigma^{; \epsilon}+\frac{2}{\epsilon} R^{\alpha}{ }_{\delta}^{\beta}{ }_{\epsilon} \sigma^{; \gamma} \dot{z}^{\delta} \dot{z}^{\epsilon}-\frac{2}{\epsilon} \dot{z}^{(\alpha} R^{\beta)}{ }_{\delta \epsilon \zeta} \dot{z}^{\gamma} \dot{z}^{\delta} \sigma^{; \epsilon} \dot{z}^{\zeta}-\frac{2}{3 \epsilon} \dot{z}^{\alpha} \dot{z}^{\beta} R_{\delta \epsilon \zeta}^{\gamma} \dot{z}^{\delta} \sigma^{; \epsilon} \dot{z}^{\zeta} \\
& \left.\mp \int_{\mp \infty}^{\tau_{x}} d \tau^{\prime} v^{\alpha \beta}{ }_{\alpha^{\prime} \beta^{\prime}} ; \gamma\left(z\left(\tau_{x}\right), z\left(\tau^{\prime}\right)\right) \dot{z}^{\alpha^{\prime}}\left(\tau^{\prime}\right) \dot{z}^{\beta^{\prime}}\left(\tau^{\prime}\right)\right)+O\left(\epsilon^{1}, \tau_{r}^{-1}\right), \\
& \psi_{\mu \nu ; \xi \rho}(x)=2 G m \bar{g}_{\mu \alpha} \bar{g}_{\nu \beta} \bar{g}_{\xi \gamma} \bar{g}_{\rho \delta} \\
& \left(-\frac{2}{\epsilon^{3}} \kappa^{-1} \dot{z}^{\alpha} \dot{z}^{\beta}\left(g^{\gamma \delta}+\kappa^{-2} \dot{z}^{\gamma} \dot{z}^{\delta}-\frac{3}{\epsilon^{2}} \sigma^{; \gamma} \sigma^{; \delta}\right)-\frac{2}{\epsilon^{3}} \dot{z}^{\alpha} \dot{z}^{\beta} \ddot{z}^{(\gamma} \sigma^{; \delta)}-\frac{8}{\epsilon^{3}} \dot{z}^{(\alpha} \ddot{z}^{\beta)} \dot{z}^{(\gamma} \sigma^{; \delta)}\right. \\
& +\frac{2}{\epsilon} \dot{z}^{(\alpha} R^{\beta)}{ }_{\epsilon}^{\gamma \delta} \dot{z}^{\epsilon}+\frac{2}{3 \epsilon} \dot{z}^{\alpha} \dot{z}^{\beta} R^{\gamma}{ }_{\epsilon}{ }_{\zeta} \dot{z}^{\epsilon} \dot{z}^{\zeta}+\frac{4}{\epsilon} \dot{z}^{(\alpha} R^{\beta)}{ }_{\epsilon}{ }^{(\gamma}{ }_{\zeta} \dot{z}^{\delta)} \dot{z}^{\epsilon} \dot{z}^{\zeta} \\
& -\frac{2}{\epsilon} R_{\epsilon}^{\alpha \beta}\left(g^{\gamma \delta}+\dot{z}^{\gamma} \dot{z}^{\delta}-\frac{1}{\epsilon^{2}} \sigma^{; \gamma} \sigma^{; \delta}\right) \dot{z}^{\epsilon} \dot{z}^{\zeta}-\frac{4}{\epsilon^{3}} \dot{z}^{(\alpha} R^{\beta)}{ }_{\epsilon}{ }^{(\gamma}{ }_{\zeta} \sigma^{; \delta)} \dot{z}^{\epsilon} \sigma^{; \zeta} \\
& -\frac{2}{3 \epsilon^{3}} \dot{z}^{\alpha} \dot{z}^{\beta} R^{\gamma}{ }_{\epsilon \zeta}{ }^{\prime} \sigma^{; \epsilon} \sigma^{; \zeta}-\frac{4}{\epsilon^{3}} \dot{z}^{(\alpha} R^{\beta)}{ }_{\epsilon \zeta \eta} \dot{z}^{(\gamma} \sigma^{; \delta)} \dot{z}^{\epsilon} \sigma^{; \zeta} \dot{z}^{\eta}-\frac{4}{3 \epsilon^{3}} \dot{z}^{\alpha} \dot{z}^{\beta} \sigma^{;(\gamma} R^{\delta)}{ }_{\epsilon \zeta \eta} \dot{z}^{\epsilon} \sigma^{; \zeta} \dot{z}^{\eta} \\
& \left.+\frac{2}{3 \epsilon^{3}} \dot{z}^{\alpha} \dot{z}^{\beta} \dot{z}^{(\gamma} R_{\epsilon \zeta \eta}^{\delta)} \sigma^{; \epsilon} \dot{z}^{\zeta} \sigma^{; \eta}\right)+O\left(\epsilon^{0}, \frac{\tau_{r}^{-1}}{\epsilon}\right) \text {. }
\end{aligned}
$$

We note that among the terms on the right hand side of the above expressions, the terms involving the Riemann tensor will not contribute to the equation of motion, as will be shown later.

Using the above expressions, one can directly check that $\psi^{\mu \nu ; \xi}{ }_{\xi}+2 R^{\mu}{ }_{\xi}^{\nu}{ }_{\rho} \psi^{\xi \rho}=0$ is satisfied for $\epsilon \neq 0$ to the order in which we are concerned. To the contrary, the harmonic gauge condition is not automatically satisfied. We find

$$
\psi_{; \nu}^{\mu \nu}=\frac{8 G m}{\epsilon} \bar{g}_{\alpha}^{\mu} \ddot{z}^{\alpha} .
$$

Generally speaking, thus obtained metric perturbation satisfies the harmonic gauge condition only when the source energy-momentum tensor satisfies the divergence free condition, which, in the present point-like particle case, leads to 
the conclusion that the source trajectory must be a geodesic. This point is totally different from the electromagnetic counterpart, in which the Lorentz gauge condition is related to the electric charge conservation. However this does not mean the break down of our formalism. Since $\ddot{z}$ is assumed to be a higher order quantity, the missing of the harmonic condition is responsible for the neglection of the higher order perturbations.

\section{DEWITT AND BREHME'S APPROACH}

In this section, we develop our discussion in an analogous way to the electromagnetic counterpart given by DeWitt and Brehme. First we derive the 'conserved' rank two symmetric tensor. Integrating its divergence over the interior of the world tube surrounding the particle, we derive the equation of motion including the effect of the gravitational radiation reaction.

\section{A. Conservation law}

In the formalism developed by DeWitt and Brehme, crucially important was the conserved energy-momentum tensor, which consists of the matter and field contributions. In the case of gravity, the matter energy-momentum tensor is divergence free by itself in the sense of the covariant derivative with respect to the total metric, $\tilde{g}_{\mu \nu}(x)$. Thus the situation looks different. However, if we choose the background spacetime $g_{\mu \nu}(x)$, we can construct a quantity analogous to the conserved energy-momentum tensor in the electromagnetic case.

We divide the metric $\tilde{g}_{\mu \nu}(x)$ into the background and the deviation from it, $\delta g_{\mu \nu}(x)$, as

$$
\tilde{g}_{\mu \nu}(x)=g_{\mu \nu}(x)+\delta g_{\mu \nu}(x)
$$

The background metric is assumed to be a solution of the vacuum Einstein equations. We write the Einstein equations with matter source, whose degrees of freedom are represented by $\phi$ symbolically, as

$$
G^{\mu \nu}[\mathbf{g}+\delta \mathbf{g}]=8 \pi G T^{\mu \nu}[\mathbf{g}+\delta \mathbf{g}, \phi]
$$

We expand $G^{\mu \nu}[\mathbf{g}+\delta \mathbf{g}]$ and $T^{\mu \nu}[\mathbf{g}+\delta \mathbf{g}, \phi]$ with respect to $\delta g_{\mu \nu}$ as

$$
\begin{aligned}
& G^{\mu \nu}[\mathbf{g}+\delta \mathbf{g}]=G^{(0) \mu \nu}+G^{(1) \mu \nu}[\delta \mathbf{g}]+G^{(2+) \mu \nu}[\delta \mathbf{g}], \\
& T^{\mu \nu}[\mathbf{g}+\delta \mathbf{g}, \phi]=T^{(0) \mu \nu}[\phi]+T^{(1+) \mu \nu}[\delta \mathbf{g}, \phi],
\end{aligned}
$$

where the superscript $(n)$ represents the terms of the $n$th order in the metric perturbation $\delta \mathbf{g}$ and the superscript $(n+)$ represents the $n$th or higher order terms. Then the Einstein equations are rewritten as

$$
\mathcal{T}^{\mu \nu}[\delta \mathbf{g}, \phi]:=T^{(0) \mu \nu}[\phi]+T^{(1+) \mu \nu}[\delta \mathbf{g}, \phi]-\frac{1}{8 \pi G} G^{(2+) \mu \nu}[\delta \mathbf{g}]=\frac{1}{8 \pi G} G^{(1) \mu \nu}[\delta \mathbf{g}]
$$

From the $O(\delta \mathbf{g})$ terms of the contracted Bianchi identities, we find $G^{(1) \mu \nu} ; \nu[\delta \mathbf{g}]=0$ when the background is a solution of the vacuum Einstein equations. Here we note again that the semicolon means the covariant derivative with respect to the background metric. Thus we obtain the covariant conservation law,

$$
\mathcal{T}^{\mu \nu}{ }_{; \nu}[\delta \mathbf{g}, \phi]=0
$$

which is what we needed.

One can see that if the mass of the particle is small enough and so the metric perturbation $\delta g_{\mu \nu}(x)$ is negligibly small, the conserved tensor $\mathcal{T}^{\mu \nu}[\delta \mathbf{g}, \phi]$ reduces to $T^{(0) \mu \nu}[\phi]$ which is independent of $\delta \mathbf{g}$, which implies $T^{(0) \mu \nu} ; \nu[\phi]=0$. Thus, in the lowest order of the metric perturbation, one observes that the point particle moves along the background geodesic.

We shall now specify our consideration to the case in which $\delta g_{\mu \nu}(x)$ is so small that we can replace $\delta g_{\mu \nu}$ by the linear perturbation $h_{\mu \nu}$ induced by $T^{(0) \mu \nu}[\phi]$. Then the conserved tensor $\mathcal{T}^{\mu \nu}$ can be approximated as

$$
\mathcal{T}^{\mu \nu}[\delta \mathbf{g}, \phi] \approx \mathcal{T}^{\mu \nu}[\mathbf{h}, \phi]=T^{(0) \mu \nu}[\phi]+T^{(1) \mu \nu}[\mathbf{h}, \phi]-\frac{1}{8 \pi G} G^{(2) \mu \nu}[\mathbf{h}] .
$$

The 1 st and 2 nd terms on the right hand side of the equation vanish outside the matter distribution, while the 3rd term bilinear in $\mathbf{h}$ does not vanish anywhere. Therefore it may be interpreted as the gravitational contribution to the energy-momentum tensor. The 3 rd term is expressed in terms of the trace-reversed metric perturbation $\psi_{\mu \nu}$ as 


$$
\begin{aligned}
G^{(2) \mu \nu}[\mathbf{h}]=\frac{1}{2} \psi & G^{(1) \mu \nu}[\mathbf{h}] \\
-\frac{1}{2}\{ & \frac{1}{2}\left(\psi^{\mu \xi ; \rho}+\psi^{\mu \rho ; \xi}-\psi^{\xi \rho ; \mu}\right)\left(\psi_{\xi ; \rho}^{\nu}+\psi_{\rho ; \xi}^{\nu}-\psi_{\xi \rho}^{; \nu}\right) \\
& +\psi_{; \rho \xi}^{\mu \xi} \psi^{\nu \rho}+\psi_{; \rho \xi}^{\nu \xi} \psi^{\mu \rho}-\psi_{; \rho \xi}^{\mu \nu} \psi^{\rho \xi}-\frac{1}{4} \psi^{; \mu} \psi^{; \nu} \\
& \left.+\frac{1}{4} g^{\mu \nu}\left(-\psi^{\xi \rho ; \sigma} \psi_{\xi \rho ; \sigma}+2 \psi^{\xi \rho ; \sigma} \psi_{\rho \sigma ; \xi}+\frac{1}{2} \psi_{; \xi}^{; \xi} \psi_{; \xi}\right)\right\} .
\end{aligned}
$$

The derivation of the above formula is given in the Appendix B.

\section{B. World Tube Around The Particle}

Following DB, we introduce a further parameterization which distinguishes the points on the same 3-surface parameterized by $\tau$. It is defined by an implicit relation between $x$ and the 4 parameters, i.e., $\tau_{x}$, $\epsilon$, and $\Omega^{i}(i=1,2,3)$,

$$
\sigma_{; \alpha}\left(x, z\left(\tau_{x}\right)\right)=-\epsilon n_{\alpha i}\left(\tau_{x}\right) \Omega^{i},
$$

where $\sum_{i=1}^{3} \Omega^{i} \Omega^{i}=1$. Here $n_{\alpha i}\left(\tau_{x}\right)$ is a set of orthonormal basis on the 3 -hypersurface of $\tau_{x}$ at $z\left(\tau_{x}\right)$. It is defined by

$$
\begin{aligned}
n_{\alpha i}(\tau) \dot{z}^{\alpha}(\tau) & =0, \\
g^{\alpha \beta}(z(\tau)) n_{\alpha i}(\tau) n_{\beta j}(\tau) & =\delta_{i j},
\end{aligned}
$$

at some $\tau_{x}$ and Fermi-Walker transported along the trajectory. In the following, we also use $\Omega^{\alpha}:=n^{\alpha}{ }_{i} \Omega^{i}$. The world tube of the particle is defined in the same manner as in DB. We consider the 4-volume of the interior of the world tube between two 3 -surfaces of $\tau_{1}$ and $\tau_{2}$,

$$
V_{\text {tube }}=\left\{x=x\left(\tau, \epsilon, \Omega^{i}\right) ; \tau_{1} \leq \tau \leq \tau_{2}, 0 \leq \epsilon \leq \epsilon_{\text {tube }}\right\} .
$$

We define the surfaces of this volume,

$$
\begin{aligned}
\Sigma_{\text {tube }} & =\left\{x=x\left(\tau, \epsilon_{\text {tube }}, \Omega^{i}\right) ; \tau_{1} \leq \tau \leq \tau_{2}\right\}, \\
\Sigma_{c a p}(\tau) & =\left\{x=x\left(\tau, \epsilon, \Omega^{i}\right) ; 0 \leq \epsilon \leq \epsilon_{\text {tube }}\right\} .
\end{aligned}
$$

The volume measure is given by $d V=\sqrt{-g(x)} d^{4} x$. The integral measures on $\Sigma_{t u b e}$ and $\Sigma_{c a p}(\tau)$ have been already derived in DB,

$$
\begin{aligned}
\left.d \Sigma_{\mu}(x)\right|_{\Sigma_{\text {tube }}} & =\left.\left(\epsilon^{2} \kappa^{2}(x) \bar{g}_{\mu \alpha}(x, z(\tau)) \Omega^{\alpha}+O\left(\epsilon^{5}\right)\right)\right|_{\Sigma_{t u b e}} d^{2} \Omega d \tau, \\
\left.d \Sigma_{\mu}(x)\right|_{\Sigma_{c a p}(\tau)} & =\left.\frac{1}{\Delta(x, z(\tau))} \epsilon^{2} \sigma_{; \mu \alpha}(x, z(\tau)) \dot{z}^{\alpha}(\tau)\right|_{\Sigma_{c a p}(\tau)} d \epsilon d^{2} \Omega,
\end{aligned}
$$

(See Eqs. (4.35) and (4.45) of DB with $R^{\alpha \beta}(z)=0$. See also Eq. (1.51) of DB. Note that our measure is with respect to the vector, while DB defines it with respect to the vector density.).

Now integrating the null quantity $\mathcal{T}^{\mu \nu} ; \nu(x)$ over $V_{\text {tube }}$ and using the integration by part, we obtain

$$
\begin{aligned}
& 0=\int_{V_{\text {tube }}} d V \bar{g}_{\beta}^{\bar{\alpha}}\left(z(\bar{\tau}), z\left(\tau_{x}\right)\right) \bar{g}^{\beta}{ }_{\mu}\left(z\left(\tau_{x}\right), x\right) \mathcal{T}_{; \nu}^{\mu \nu}(x) \\
& =\int_{\Sigma_{t u b e}+\Sigma_{c a p}\left(\tau_{2}\right)-\Sigma_{c a p}\left(\tau_{1}\right)} d \Sigma_{\nu}(x) \bar{g}^{\bar{\alpha}}{ }_{\beta}\left(z(\bar{\tau}), z\left(\tau_{x}\right)\right) \bar{g}^{\beta}{ }_{\mu}\left(z\left(\tau_{x}\right), x\right) \mathcal{T}^{\mu \nu}(x) \\
& -\int_{V_{\text {tube }}} d V\left[\bar{g}^{\bar{\alpha}}{ }_{\beta}\left(z(\bar{\tau}), z\left(\tau_{x}\right)\right) \bar{g}^{\beta}{ }_{\mu}\left(z\left(\tau_{x}\right), x\right)\right]_{; \nu} \mathcal{T}^{\mu \nu}(x),
\end{aligned}
$$

where $\bar{\tau}=\left(\tau_{1}+\tau_{2}\right) / 2$. The reason why $\bar{g}_{\beta}{ }_{\beta}\left(z(\bar{\tau}), z\left(\tau_{x}\right)\right) \bar{g}^{\beta}{ }_{\mu}\left(z\left(\tau_{x}\right), x\right)$ is multiplied is to make the integrand to be a vector at $z(\bar{\tau})$ and to behave as a scalar with respect to $x$, so that the integration is done in the covariant manner. 


\section{Gravitational Radiation Damping}

To evaluate the second and third lines of Eq. (3.17), we have to put an assumption on the matter configuration. We take the tube radius $\epsilon_{\text {tube }}$ so that the tube is in the external zone. Therefore, the metric perturbation on the tube surface $\Sigma_{\text {tube }}$ can be approximated by that induced by a point-like particle given in Eq. (2.20). Noting that no contribution of matter to $\mathcal{T}^{\mu \nu}[\mathbf{h}, \phi]$ exists there, $\mathcal{T}^{\mu \nu}[\mathbf{h}, \phi]$ can be computed as

$$
\begin{gathered}
\mathcal{T}^{\mu \nu}[\mathbf{h}, \phi]=\frac{G m^{2}}{4 \pi} \bar{g}^{\mu}{ }_{\alpha}\left(x, z\left(\tau_{x}\right)\right) \bar{g}^{\nu}{ }_{\beta}\left(x, z\left(\tau_{x}\right)\right) \\
\left\{\frac{1}{\epsilon^{4}}\left(-4 \dot{z}^{\alpha} \dot{z}^{\beta}+\Omega^{\alpha} \Omega^{\beta}-\frac{1}{2} g^{\alpha \beta}\right)+\frac{1}{\epsilon^{3}}\left(-4 \dot{z}^{\alpha} \dot{z}^{\beta} \ddot{z}^{\gamma} \Omega_{\gamma}-7 \ddot{z}^{(\alpha} \Omega^{\beta)}+\frac{7}{2} g^{\alpha \beta} \ddot{z}^{\gamma} \Omega_{\gamma}\right)\right. \\
-\frac{1}{\epsilon^{2}}\left(8 \dot{z}^{(\alpha} V^{\beta)}{ }_{\gamma \delta} \dot{z}^{(\gamma} \Omega^{\delta)}-4 V_{\gamma \delta}{ }^{(\alpha} \dot{z}^{\beta)} \dot{z}^{\gamma} \Omega^{\delta}+2 g^{\alpha \beta} V_{\gamma \delta \epsilon} \dot{z}^{\gamma} \Omega^{\delta} \dot{z}^{\epsilon}-4 \Omega^{(\alpha} V^{\beta)}{ }_{\gamma \delta} \dot{z}^{\gamma} \dot{z}^{\delta}\right. \\
\left.\left.+2 V_{\gamma \delta}{ }^{(\alpha} \Omega^{\beta)} \dot{z}^{\gamma} \dot{z}^{\delta}-g^{\alpha \beta} V_{\gamma \delta \epsilon} \dot{z}^{\gamma} \dot{z}^{\delta} \Omega^{\epsilon}+\Omega^{(\alpha} V^{\beta)}-\frac{1}{2} g^{\alpha \beta} V_{\gamma} \Omega^{\gamma}\right)\right\} \\
+\mathcal{T}_{R}^{\mu \nu}+O\left(\dddot{z}, \frac{\tau_{r}^{-1}}{\epsilon^{2}}, \frac{1}{\epsilon}\right),
\end{gathered}
$$

where $z, \dot{z}, \ddot{z}$ are evaluated at $\tau=\tau_{x}$, and we have defined

$$
\begin{aligned}
V_{\mu \nu \xi}(x) & :=\mp \int_{\mp \infty}^{\tau_{x}} d \tau^{\prime} v_{\mu \nu \alpha^{\prime} \beta^{\prime} ; \xi}\left(x, z\left(\tau^{\prime}\right)\right) \dot{z}^{\alpha^{\prime}}\left(\tau^{\prime}\right) \dot{z}^{\beta^{\prime}}\left(\tau^{\prime}\right), \\
V_{\mu}(x) & :=\mp \int_{\mp \infty}^{\tau_{x}} d \tau^{\prime} v^{\nu}{ }_{\nu \alpha^{\prime} \beta^{\prime} ; \mu}\left(x, z\left(\tau^{\prime}\right)\right) \dot{z}^{\alpha^{\prime}}\left(\tau^{\prime}\right) \dot{z}^{\beta^{\prime}}\left(\tau^{\prime}\right) .
\end{aligned}
$$

Here we have denoted the terms which contain the Riemann tensor by $\mathcal{T}_{R}^{\mu \nu}$, which is at most of $O\left(1 / \epsilon^{2}\right)$. It is not necessary to write them down explicitly because they do not affect the equation of motion, as will be shown later.

Noting that the terms containing odd number of $\Omega$ 's in the integrand vanish when $d^{2} \Omega$-integration is done, and that $\int d \Omega^{2} \Omega^{\alpha} \Omega^{\beta}=\frac{4 \pi}{3}\left(g^{\alpha \beta}+\dot{z}^{\alpha} \dot{z}^{\beta}\right)$, the integration over the surface of the tube $\Sigma_{\text {tube }}$ for small $\delta \tau:=\tau_{2}-\tau_{1}$ is evaluated as

$$
\begin{aligned}
& \int_{\Sigma_{\text {tube }}} d \Sigma_{\nu}(x) \bar{g}^{\bar{\alpha}}{ }_{\beta}\left(z(\bar{\tau}), z\left(\tau_{x}\right)\right) \bar{g}_{\mu}^{\beta}\left(z\left(\tau_{x}\right), x\right) \mathcal{T}^{\mu \nu}[\mathbf{h}](x) \\
& =G m^{2}\left\{\left(-\frac{7}{2 \epsilon_{\text {tube }}} \ddot{z}^{\bar{\alpha}}-\frac{2}{3} \dot{z}^{\bar{\alpha}} V_{\bar{\beta} \bar{\gamma} \bar{\delta}} \dot{z}^{\bar{\beta}} \dot{z}^{\bar{\gamma}} \dot{z}^{\bar{\delta}}-\frac{2}{3} \dot{z}^{\bar{\alpha}} V_{\bar{\beta}} \dot{z}^{\bar{\beta}}\right.\right. \\
& \left.\left.\quad+\left(V_{\bar{\beta} \bar{\gamma}}^{\bar{\alpha}}+V^{\bar{\alpha}}{ }_{\bar{\gamma} \bar{\beta}}-V_{\bar{\beta} \bar{\gamma}}{ }^{\bar{\alpha}}\right) \dot{z}^{\bar{\beta}} \dot{z}^{\bar{\gamma}}-\frac{1}{2} V^{\bar{\alpha}}\right)(\bar{\tau})+O\left(\tau_{r}^{-1}, \epsilon_{\text {tube }}\right)\right\} \delta \tau+O\left(\delta \tau^{2}\right) .
\end{aligned}
$$

The surface integration over the cap $\Sigma_{c a p}$ requires the knowledge of the metric in the internal zone. We assume that the matter configuration and the metric is not perturbed much and is kept spherically symmetric inside the radius $\epsilon_{0}(\gg G m)$ to sufficient accuracy. In other words, locally the particle behaves as if it were an isolated object. Mathematically, we assume that the cap integration for $\epsilon \leq \epsilon_{0}$, which in an unrigorous manner defines the particle momentum $p_{\epsilon_{0}}^{\alpha}(\tau)$, is proportional to $\dot{z}^{\alpha}(\tau)$,

$$
\begin{aligned}
\int_{\Sigma_{c a p}(\tau), \epsilon<\epsilon_{0}} d \Sigma_{\nu}(x) \bar{g}_{\beta}^{\bar{\alpha}}\left(z(\bar{\tau}), z\left(\tau_{x}\right)\right) \bar{g}^{\beta}{ }_{\mu}\left(z\left(\tau_{x}\right), x\right) \mathcal{T}^{\mu \nu}[\mathbf{h}](x) \\
=m\left(\epsilon_{0}, \tau\right) \bar{g}^{\bar{\alpha}}{ }_{\beta}(z(\bar{\tau}), z(\tau)) \dot{z}^{\beta}(\tau)+G m^{2} O\left(m, \tau_{r}^{-1}, \epsilon_{\text {tube }}\right) .
\end{aligned}
$$

We call the matter which satisfies this assumption as an ideal point-like particle.

Outside the radius $\epsilon_{0}$, the metric is approximated by that induced by a point-like particle, $(2.20)$. Then the cap integration for $\epsilon_{0} \leq \epsilon \leq \epsilon_{\text {tube }}$ is evaluated by using Eq. (3.18) to give

$$
\int_{\Sigma_{c a p}(\tau), \epsilon>\epsilon_{0}} d \Sigma_{\nu}(x) \bar{g}_{\beta}^{\bar{\alpha}}\left(z(\bar{\tau}), z\left(\tau_{x}\right)\right) \bar{g}_{\mu}^{\beta}\left(z\left(\tau_{x}\right), x\right) \mathcal{T}^{\mu \nu}[\mathbf{h}](x)
$$




$$
=-\frac{7}{2} G m^{2}\left[\left(\frac{1}{\epsilon_{0}}-\frac{1}{\epsilon_{\text {tube }}}\right) \bar{g}^{\bar{\alpha}}{ }_{\beta}(z(\bar{\tau}), z(\tau)) \dot{z}^{\beta}(\tau)+O\left(\tau_{r}^{-1}, \epsilon_{t u b e}\right)\right] .
$$

Thus setting

$$
m\left(\epsilon_{0}, \tau\right)=m(\tau)+\frac{7}{2} \frac{G m^{2}}{\epsilon_{0}}
$$

we obtain

$$
\begin{aligned}
\int_{\Sigma_{c a p}\left(\tau_{2}\right)-\Sigma_{c a p}\left(\tau_{1}\right)} & d \Sigma_{\nu}(x) \bar{g}^{\bar{\alpha}}{ }_{\beta}\left(z(\bar{\tau}), z\left(\tau_{x}\right)\right) \bar{g}^{\beta}{ }_{\mu}\left(z\left(\tau_{x}\right), x\right) \mathcal{T}^{\mu \nu}[\mathbf{h}](x) \\
= & {\left[\left\{m(\bar{\tau})+\frac{7}{2} \frac{G m^{2}}{\epsilon_{\text {tube }}}\right\} \ddot{z}^{\bar{\alpha}}(\bar{\tau})+\dot{m}(\bar{\tau}) \dot{z}^{\bar{\alpha}}(\bar{\tau})\right] \delta \tau+O\left(\delta \tau^{2}\right) . }
\end{aligned}
$$

Finally, we consider the remaining volume integral $\int_{V_{t u b e}} d V\left[\bar{g}^{\bar{\alpha}}{ }_{\beta} \bar{g}_{\mu}\right]_{; \nu} \mathcal{T}^{\mu \nu}$. Since $\left[\bar{g}^{\bar{\alpha}}{ }_{\beta} \bar{g}^{\alpha}{ }_{\mu}\right]_{; \nu} \approx \epsilon \Omega$, again we expect that the integration for $\epsilon<\epsilon_{0}$ vanishes for the ideal point-like particle to sufficient accuracy. The integration for $\epsilon>\epsilon_{0}$ is at most of $O\left(G m^{2} \epsilon_{0} \delta \tau\right)$. Thus we may neglect the contribution from the volume integral.

Putting all results together, we obtain the equation of motion,

$$
\begin{aligned}
m(\tau) \ddot{z}^{\alpha}+\dot{m}(\tau) \dot{z}^{\alpha}=G m^{2} & \left\{\frac{2}{3} \dot{z}^{\alpha} V_{\beta \gamma \delta} \dot{z}^{\beta} \dot{z}^{\gamma} \dot{z}^{\delta}+\frac{2}{3} \dot{z}^{\alpha} V_{\beta} \dot{z}^{\beta}\right. \\
& \left.-\left(V^{\alpha}{ }_{\beta \gamma}+V^{\alpha \beta}{ }_{\gamma \beta}-V_{\beta \gamma}{ }^{\alpha}\right) \dot{z}^{\beta} \dot{z}^{\gamma}+\frac{1}{2} V^{\alpha}+O\left(\epsilon_{\text {tube }}, \tau_{r}^{-1}\right)\right\} .
\end{aligned}
$$

The leading contributions from the terms proportional to the Riemann tensor which we have neglected are at most of the zeroth order in $\epsilon_{t u b e}$ (or $\epsilon_{0}$ ). As they should be vectors, they must take the form $G m^{2} R^{\mu}{ }_{\nu \rho \sigma} \dot{z}^{\nu} \dot{z}^{\rho} \dot{z}^{\sigma}$ or $G m^{2} R^{\mu}{ }_{\nu \rho \sigma} g^{\nu \rho} \dot{z}^{\sigma}$ by means of the dimensional argument. Thus all the leading terms disappear and the remaining terms become higher order in $\epsilon_{\text {tube }}$ (or $\epsilon_{0}$ ).

Now we consider the normalization condition $g_{\alpha \beta}(z(\tau)) \dot{z}^{\alpha}(\tau) \ddot{z}^{\beta}(\tau)=0$. This gives

$$
\dot{m}(\tau)=G m^{2}\left\{\frac{5}{3} V_{\beta \gamma \delta} \dot{z}^{\beta} \dot{z}^{\gamma} \dot{z}^{\delta}+\frac{1}{6} V_{\beta} \dot{z}^{\beta}\right\},
$$

and it can be integrated as

$$
m(\tau)=m\left\{1+\left(\frac{5}{6} \dot{z}^{\beta} \dot{z}^{\gamma}+\frac{1}{12} g^{\beta \gamma}\right) \psi_{(v) \beta \gamma}(z(\tau))\right\}
$$

where we have defined

$$
\psi_{(v) \mu \nu}(x):=\mp 2 G m \int_{\mp \infty}^{\tau_{x}} d \tau^{\prime} v_{\mu \nu \alpha^{\prime} \beta^{\prime}}\left(x, z\left(\tau^{\prime}\right)\right) \dot{z}^{\alpha^{\prime}}\left(\tau^{\prime}\right) \dot{z}^{\beta^{\prime}}\left(\tau^{\prime}\right),
$$

which is the part of the trace-reversed metric perturbation due to the $v_{\mu \nu \alpha \beta}(x, z)$ term (i.e, the so-called tail term) in the Green function (see Eq. (2.20)). Since $\psi_{(v) \beta \gamma}$ is expected to be of $O(G m)$, Eq. (3.28) tells us that we can consistently replace $m(\tau)$ by $m$ in Eq. (3.26) except for the term, $\dot{m}(\tau) \dot{z}^{\alpha}$.

Substituting Eq. (3.27) into Eq. (3.26), we finally obtain

$$
\begin{aligned}
m \ddot{z}^{\alpha}(\tau)=-m( & \frac{1}{2} \dot{z}^{\alpha} \dot{z}^{\beta} \dot{z}^{\gamma} \dot{z}^{\delta}+g^{\alpha \beta}(z) \dot{z}^{\gamma} \dot{z}^{\delta}-\frac{1}{2} g^{\alpha \delta}(z) \dot{z}^{\beta} \dot{z}^{\gamma} \\
& \left.-\frac{1}{4} \dot{z}^{\alpha} g^{\beta \gamma}(z) \dot{z}^{\delta}-\frac{1}{4} g^{\alpha \delta}(z) g^{\beta \gamma}(z)\right)(\tau) \psi_{(v) \beta \gamma ; \delta}(z(\tau)),
\end{aligned}
$$

where we have also used the relation $V_{\alpha \beta \gamma}(z(\tau))=\psi_{(v) \alpha \beta ; \gamma}(z(\tau))$ which follows from Eq. (2.11). If we impose the physical boundary condition with no-incoming waves from the past null infinity, we should take the upper sign (i.e., the retarded boundary condition) of Eq. (3.29) for $\psi_{(v) \beta \gamma}$. The meaning of this equation is discussed in section 5. 


\section{MATCHED ASYMPTOTIC EXPANSION}

In this section we give an alternative derivation of the equation of motion obtained in the previous section in a more rigorous way by using the matched asymptotic expansion technique.

\section{A. Matching Scheme}

To begin with, we state the general concept of the asymptotic matching. We first prepare the metrics in both internal and external zones by using different schemes. In the internal zone, we expect that the metric can be described by using the black hole perturbation. Namely, we assume that the particle is represented by a Schwarzschild black hole in the lowest order of approximation. In the present case, the perturbation is caused by the tidal effects of the curvature of the spacetime in which the particle travels. We call this construction of the metric the internal scheme. In order to make this scheme valid, the linear extension of the internal zone around the particle must be much smaller than the background curvature scale. We use the coordinate $\left\{X^{a}\right\}=\left\{T, X^{i}\right\} \quad(a=0,1,2,3 ; i=1,2,3)$ for the internal scheme and $|X|\left(:=\sqrt{X^{i} X^{i}}\right)$ is assumed to represent the physical distance scale (in this section, we adopt the Minkowskian summation rule on $a, b, \cdots$, and the Kronecker summation rule on $i, j, \cdots$ over the repeated indices). In the external zone, as discussed in the previous sections, we expect that the metric is represented by the perturbations induced by a particle on a given background spacetime. We call this construction of the metric the external scheme. We require that the metrics obtained in both schemes be matched in the matching region of both zones, by considering the coordinate transformation between the internal and external zones. Safely, we may assume the existence of the matching region as long as $G m \ll L \sim 1$. we set the matching radius at $|X| \sim(G m L)^{1 / 2}$ by using the spatial coordinates of the internal scheme, $X^{i}$. Then, writing down the metric in the internal scheme, we have two independent small parameters $|X| / L$ and $G m /|X|$ in the matching region. The power expansion with respect to these two small parameters allows us to consider the matching order by order.

First we consider the expansion of the internal scheme. Recalling that the perturbation in the internal zone is induced by the external curvature which is characterized by the length $L$, the metric can be expanded in powers of $|X| / L$ as

$$
\tilde{g}_{a b}(X)=\stackrel{(0)}{H}_{a b}(X)+\frac{1}{L} \stackrel{(1)}{H}_{a b}(X)+\frac{1}{L^{2}} \stackrel{(2)}{H}_{a b}(X)+\cdots,
$$

where $\stackrel{(0)}{H}_{a b}(X)$ represents the unperturbed black hole metric. We expect that $\stackrel{(1)}{H}_{a b}(X)$ will be given by the standard linear perturbation of the black hole. Later, we find that the matching condition requires that $\stackrel{(1)}{H} a b(X)$ should vanish, which is consistent with the notion that the spacetime curvature is of $O\left(1 / L^{2}\right)$. Thus the standard black hole (2)

perturbation theory applies up to $\mathrm{H}_{a b}(X)$. As is known well, the linear perturbations of the Schwarzschild black hole 8] can be decomposed by using the tensor harmonics, which are classified by the total angular momentum, $J$. The monopole mode $(J=0)$ corresponds to the mass perturbation. Thus we may set this mode to vanish since it is natural to suppose that the change of mass due to the radiation reaction is small. The dipole modes $(J=1)$ are related to the translation and rotation. The translation modes are purely gauge and thus we set them to vanish to fix the center of the black hole. As we are considering a non-rotating black hole, we also set the rotational modes to vanish. In general, the higher modes contain gauge degrees of freedom as well as the physical ones. However, for these higher modes, we do not give any principle to fix the gauge for the moment. Further we expand the metric with respect to $G m /|X|$ as

$$
\begin{aligned}
& \stackrel{(0)}{H}_{a b}(X)=\eta_{a b}+G m \underset{(1)}{\stackrel{(0)}{H}} a b(X)+(G m)^{2} \stackrel{(0)}{H}_{(2)} a b(X)+\cdots, \\
& \frac{1}{L} \stackrel{(1)}{H}_{a b}(X)=\frac{1}{L} \stackrel{(1)}{H}_{(0)} a b(X)+\frac{G m}{L} \stackrel{(1)}{H}_{(1)} a b(X)+\frac{(G m)^{2}}{L} \stackrel{(1)}{H}_{(2)} a b(X)+\cdots, \\
& \frac{1}{L^{2}} \stackrel{(2)}{H}_{a b}(X)=\frac{1}{L^{2}} \stackrel{(2)}{H}_{(0)} a b(X)+\frac{G m}{L^{2}} \underset{(1)}{\stackrel{(2)}{H}} a b(X)+\frac{(G m)^{2}}{L^{2}}{\underset{(2)}{H}}_{a b}(X)+\cdots .
\end{aligned}
$$

Note that, from the definitions of the expansion parameters, the component of the metric behaves as

$$
\stackrel{(m)}{H} a b \sim|X|^{(m-n)}
$$

In order to write down the external metric in terms of the internal coordinates, we consider the coordinate transformation from $x$ to $\left(T, X^{i}\right)$ given in the form, 


$$
\sigma_{; \alpha}(x, z(T))=-\mathcal{F}_{\alpha}(T, X) .
$$

We assume $X^{i}=0$ corresponds to the center of the particle, $x^{\alpha}=z^{\alpha}(T)$, hence $\mathcal{F}_{\alpha}=0$ at $X^{i}=0$. We suppose that the right hand side of Eq. (4.4) can be expanded in positive powers of $X^{i}$ as

$$
\mathcal{F}_{\alpha}(T, X)=f_{\alpha i}(T) X^{i}+\frac{1}{2} f_{\alpha i j}(T) X^{i} X^{j}+\frac{1}{3 !} f_{\alpha i j k}(T) X^{i} X^{j} X^{k}+\cdots .
$$

Although it is possible that more complicated terms such as $X^{i} X^{j} /|X|$ may appear, we simply ignore these kinds of terms. We shall find it is consistent within the order of the approximation to which we are going to develop our consideration below. Here $f_{\alpha i_{1} \cdots i_{n}}(T)$ is totally symmetric for $i_{1} \cdots i_{n}$ and is at most of $O\left(L^{-(n-1)}\right)$. Using Eqs. (A9) and (A11) in Appendix A, the total derivative of Eq. (4.4) gives the important relation,

$$
\begin{aligned}
\bar{g}_{\mu}^{\alpha}(z(T), x) d x^{\mu}= & \left(\frac{d z^{\alpha}}{d T}(T)+\frac{D f^{\alpha}{ }_{i}}{d T}(T) X^{i}+\frac{1}{2} \frac{D f^{\alpha}{ }_{i j}}{d T}(T) X^{i} X^{j}\right. \\
& \left.+\frac{1}{2} R^{\alpha}{ }_{\beta \gamma \delta}(z(T)) f^{\beta}{ }_{i}(T) \frac{d z^{\gamma}}{d T}(T) f^{\delta}{ }_{j}(T) X^{i} X^{j}+O\left(|X|^{3}\right)\right) d T \\
& +\left(f^{\alpha}{ }_{i}+f^{\alpha}{ }_{i j}(T) X^{j}+\frac{1}{2} f^{\alpha}{ }_{i j k}(T) X^{j} X^{k}\right. \\
& \left.+\frac{1}{6} R^{\alpha}{ }_{\beta \gamma \delta}(z(T)) f^{\beta}{ }_{j}(T) f^{\gamma}{ }_{i}(T) f^{\delta}{ }_{k}(T) X^{j} X^{k}+O\left(|X|^{3}\right)\right) d X^{i}
\end{aligned}
$$

Then, with the aid of Eqs. (4.4) and (4.6), the external metric $\tilde{g}_{\mu \nu}(x)$ can be transformed into that written in terms of the internal coordinates by the relation,

$$
\tilde{g}_{a b}(X) d X^{a} d X^{b}=\tilde{g}_{\mu \nu}(x) d x^{\mu} d x^{\nu} .
$$

Generally, as the external metric can be expanded by $G m /|X|$, we write it as

$$
\tilde{g}_{a b}(X)=g_{a b}(X)+G m \underset{(1)}{h a b}(X)+(G m)^{2} \underset{(2)}{h_{a b}}(X)+\cdots .
$$

Then $G m h_{a b}(X)$ can be recognized as the linear perturbation on the background $g_{a b}(X)$. Further we expand it (1) with respect to $|X| / L$ as

$$
\begin{aligned}
& g_{a b}(X)=\underset{(0)}{\stackrel{(0)}{h}} a b(X)+\frac{1}{L} \underset{(0)}{\stackrel{(1)}{h} a b}(X)+\frac{1}{L^{2}} \underset{(0)}{\stackrel{(2)}{h} a b}(X)+\cdots,
\end{aligned}
$$

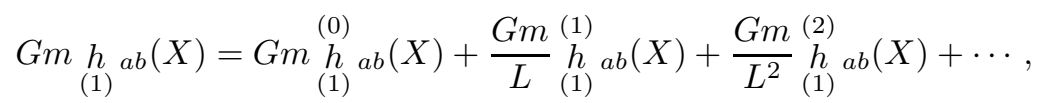

$$
\begin{aligned}
& (G m)^{2} \underset{(2)}{h} a b(X)=(G m)^{2} \underset{(2)}{\stackrel{(0)}{h}} a b(X)+\frac{(G m)^{2}}{L} \underset{(2)}{\stackrel{(1)}{h}} a b(X)+\frac{(G m)^{2}}{L^{2}} \underset{(2)}{\stackrel{(2)}{h}} a b(X)+\cdots .
\end{aligned}
$$

As before,

$$
\stackrel{(m)}{h} a b \sim|X|^{(m-n)}
$$

For brevity, we call $\underset{(n)}{\stackrel{(m)}{h}}$ ab or $\underset{(n)}{\stackrel{(m)}{H}}$ ab the $\left(\begin{array}{c}m \\ n\end{array}\right)$ component and the matching condition for them as the $\left(\begin{array}{c}m \\ n\end{array}\right)$ matching. In the matching region $\left(|X| \sim(G m L)^{1 / 2}\right)$, the $\left(\begin{array}{c}m \\ n\end{array}\right)$ component is of $O\left((G m / L)^{(m+n) / 2}\right)$. The matching condition requires that all the corresponding terms in Eqs. (4.2) and (4.9) should be identical. Then what we have to do is to equate the terms of the same power in $|X|$ to desired accuracy. Thus the condition for the $\left(\begin{array}{c}m \\ n\end{array}\right)$ matching is

$$
\sum_{\substack{m^{\prime}-n^{\prime}=m-n \\ m^{\prime} \leq m}} \frac{(G m)^{n^{\prime}}}{L^{m^{\prime}}} \underset{\substack{\left(m^{\prime}\right) \\\left(n^{\prime}\right)}}{h}=\sum_{\substack{m^{\prime}-n^{\prime}=m-n \\ m^{\prime} \leq m}} \frac{(G m)^{n^{\prime}}}{L^{m^{\prime}}} \underset{\left(n^{\prime}\right)}{\stackrel{\left(m^{\prime}\right)}{H}} a b+O\left(\frac{(G m)^{n+1}}{L^{m+1}}|X|^{(m-n)}\right) .
$$




\section{B. Geodesic; $\left(\begin{array}{l}0 \\ 0\end{array}\right)$ and $\left(\begin{array}{l}1 \\ 0\end{array}\right)$ Matching}

We begin with the $\left(\begin{array}{l}0 \\ 0\end{array}\right)$ and $\left(\begin{array}{l}1 \\ 0\end{array}\right)$ matchings, which are of $O\left((G m / L)^{0}\right)$ and of $O\left((G m / L)^{1 / 2}\right)$ in the matching region, respectively. First we consider the external scheme. In these matchings the external metric is the background itself. Here, the necessary order of expansion in $|X|$ is $O(|X|)$. Since $g_{\mu \nu}(x) d x^{\mu} d x^{\nu}=g_{\alpha \beta}(z) \bar{g}^{\alpha}{ }_{\mu}(z, x) \bar{g}^{\beta}{ }_{\nu}(z, x) d x^{\mu} d x^{\nu}($ see Eq. (1.33) of DB), we get

$$
\begin{aligned}
g_{\mu \nu}(x) d x^{\mu} d x^{\nu}= & \left(\left(\frac{d z}{d T}\right)^{2}(T)+2 \frac{d z^{\alpha}}{d T}(T) \frac{D f_{\alpha i}}{d T}(T) X^{i}+O\left(\frac{|X|^{2}}{L^{2}}\right)\right) d T^{2} \\
& +2\left(\frac{d z^{\alpha}}{d T}(T) f_{\alpha i}(T)+\frac{d z^{\alpha}}{d T}(T) f_{\alpha i j}(T) X^{j}+f^{\alpha}{ }_{i}(T) \frac{D f_{\alpha j}}{d T}(T) X^{j}+O\left(\frac{|X|^{2}}{L^{2}}\right)\right) d T d X^{i} \\
& +\left(f_{i}^{\alpha}(T) f_{\alpha j}(T)+2 f^{\alpha}{ }_{i}(T) f_{\alpha j k}(T) X^{k}+O\left(\frac{|X|^{2}}{L^{2}}\right)\right) d X^{i} d X^{j}
\end{aligned}
$$

Comparing the above with Eq. (4.9) and looking at the dependence on $X$, one can readily extract out $\stackrel{(0)}{h} a b$ and $\stackrel{(1)}{h} a b$ to the lowest order in $G m / L$.

Next we consider the internal scheme. The $\left(\begin{array}{l}0 \\ 0\end{array}\right)$-component is trivially given by the flat Minkowski metric. To know the $\left(\begin{array}{l}1 \\ 0\end{array}\right)$-component of the internal scheme, it is better to consider all the $\left(\begin{array}{l}1 \\ n\end{array}\right)$-components at the same time. Namely we consider the linear perturbation of the black hole $\stackrel{(1)}{H}$ ab. For this purpose, we consider the decomposition of the linear perturbation in terms of the tensor harmonics as discussed in Appendix C. Since the timescale associated with the perturbation should be of the order of the background curvature scale $L$, it is much larger than the matching radius $(G m L)^{1 / 2}$. Therefore the perturbation may be regarded as static. It is known that all the physical static perturbations regular on the black hole horizon behave as $\sim|X|^{J}$ asymptotically, where $J$ is the angular momentum eigenvalue. However, in $\stackrel{(1)}{H}_{a b}$, there exists no term which behaves as $\sim|X|^{m},(m \geq 2)$. Hence, except for gauge degrees of freedom, $\stackrel{(1)}{H}_{a b}$ contains only $J=0,1$ modes. As mentioned before, we set the perturbation of $J=0,1$ modes to vanish. Thus we conclude that we may set $\stackrel{(1)}{H} a b=0$.

The $\left(\begin{array}{l}0 \\ 0\end{array}\right)$ matching becomes

$$
\begin{aligned}
& -1=\left(\frac{d z}{d T}\right)^{2}(T)+O\left(\frac{G m}{L}\right), \quad(T T) \text {-component } \\
& 0=\frac{d z^{\alpha}}{d T}(T) f_{\alpha i}(T)+\left(\frac{G m}{L}\right), \quad(T i) \text {-components, } \\
& \delta_{i j}=f^{\alpha}{ }_{i}(T) f_{\alpha j}(T)+\left(\frac{G m}{L}\right), \quad(i j) \text {-components. }
\end{aligned}
$$

Equations (4.14) and (4.15) indicate that $f^{\alpha i}(T)$ are spatial triad basis along the orbit, i.e.,

$$
f_{k}^{\alpha}(T) f_{k}^{\beta}(T)=g^{\alpha \beta}(z(T))+\frac{d z^{\alpha}}{d T}(T) \frac{d z^{\beta}}{d T}(T)+O\left(\frac{G m}{L}\right) .
$$

The $\left(\begin{array}{l}1 \\ 0\end{array}\right)$ matching becomes

$$
\begin{aligned}
& 0=2 \frac{d z^{\alpha}}{d T}(T) \frac{D f_{\alpha i}}{d T}(T) X^{i}+\left(\frac{G m}{L} \frac{|X|}{L}\right), \quad(T T) \text {-component, } \\
& 0=\frac{d z^{\alpha}}{d T}(T) f_{\alpha i j}(T) X^{j}+f^{\alpha}{ }_{i}(T) \frac{D f_{\alpha j}}{d T}(T) X^{j}+\left(\frac{G m}{L} \frac{|X|}{L}\right), \quad(T i) \text {-components, } \\
& 0=2 f_{\alpha(i}(T) f_{j) k}^{\alpha}(T) X^{k}+\left(\frac{G m}{L} \frac{|X|}{L}\right), \quad(i j) \text {-components. }
\end{aligned}
$$

Then the covariant T-derivative of Eq. (4.13) and that of Eq. (4.14) with Eq. (4.17) result in the background geodetic motion, 


$$
\frac{D}{d T}\left(\frac{d z^{\alpha}}{d T}\right)(T)=O\left(\frac{G m}{L} \frac{1}{L}\right)
$$

One can see that the internal time coordinate $T$ becomes a proper time of the orbit from Eq. (4.13) in the lowest order in $G m / L$. In the same manner, Eq. (4.17) and the covariant $T$-derivative of Eq. (4.15) with (ij)-antisymmetric part of Eq. (4.18) give the geodetic transport of the triad $f^{\alpha}{ }_{i}(T)$,

$$
\frac{D}{d T} f_{i}^{\alpha}(T)=O\left(\frac{G m}{L} \frac{1}{L}\right)
$$

Further, from Eqs. (4.18) and (4.19), we can see

$$
f^{\alpha}{ }_{i j}(T)=O\left(\frac{G m}{L} \frac{1}{L}\right)
$$

\section{Hypersurface Condition; $\left(\begin{array}{l}2 \\ 0\end{array}\right)$ Matching}

We now proceed to the $\left(\begin{array}{l}2 \\ 0\end{array}\right)$ matching, in which the external metric is still given by the background but there appear non-trivial perturbations of the internal scheme. Although it is of $O(G \mathrm{~m} / L)$ in the matching region and $O\left((G m / L)^{1 / 2}\right)$ higher than the remaining $\left(\begin{array}{l}0 \\ 1\end{array}\right)$-component, we consider it first for the reason which will be clarified below.

In order to obtain $\underset{(0)}{\stackrel{(2)}{h}} a b$, we expand the external metric in terms of the internal coordinates up to $O\left(|X|^{2}\right)$, i.e., we have to go one order higher than Eq. (4.12). Then the $\left(\begin{array}{l}2 \\ 0\end{array}\right)$ matching becomes

$$
\begin{aligned}
\frac{1}{L^{2}} \stackrel{(2)}{H}_{(0)}= & R_{\alpha \beta \gamma \delta}(z(T)) \frac{d z^{\alpha}}{d T}(T) f^{\beta}{ }_{i}(T) \frac{d z^{\gamma}}{d T}(T) f^{\delta}{ }_{j}(T) X^{i} X^{j}+O\left(\frac{G m}{L} \frac{|X|^{2}}{L^{2}}\right), \\
& (T T) \text {-component, } \\
\frac{1}{L^{2}} \stackrel{(2)}{H}_{(0)}= & \frac{1}{2} \frac{d z^{\alpha}}{d T}(T) f_{\alpha i j k}(T) X^{j} X^{k}+\frac{2}{3} R_{\alpha \beta \gamma \delta}(z(T)) \frac{d z^{\alpha}}{d T}(T) f^{\beta}{ }_{j}(T) f^{\gamma}{ }_{i}(T) f^{\delta}{ }_{k}(T) X^{j} X^{k}+O\left(\frac{G m}{L} \frac{|X|^{2}}{L^{2}}\right), \\
& (T i) \text {-component, } \\
\frac{1}{L^{2}} \underset{(0)}{{ }_{(0)}^{H}}{ }_{i j}= & f_{\alpha(i}(T) f^{\alpha}{ }_{j) k l}(T) X^{k} X^{l}+\frac{1}{3} R_{\alpha \beta \gamma \delta}(z(T)) f^{\alpha}{ }_{i}(T) f^{\beta}{ }_{k}(T) f^{\gamma}{ }_{j}(T) f^{\delta}{ }_{l}(T) X^{k} X^{l}+O\left(\frac{G m}{L} \frac{|X|^{2}}{L^{2}}\right),
\end{aligned}
$$

(ij)-component,

where Eqs. (4.21) and (4.22) have been used to simplify the expressions. Since there appear terms which describe $J=2$ perturbations of the internal scheme, the $\left(\begin{array}{l}2 \\ 0\end{array}\right)$ matching does not determine $f^{\alpha}{ }_{i j k}$ without specifying the gauge condition of the internal scheme. Here we fix it as follows.

As before, we first set $J=0,1$ modes of $\underset{(0)}{\stackrel{(2)}{H}}$ ab equal to zero. Applying the discussion given in Appendix C, we find the $(T T)$-component does not contain $J=0,1$ modes. As for the $(T i)$-component, it contains no $J=0$ mode but the $J=1$ mode is present. Setting it to vanish to the lowest order in $G m / L$, we find

$$
\frac{d z^{\alpha}}{d T}(T) f_{\alpha i k k}(T)=O\left(\frac{G m}{L} \frac{1}{L^{3}}\right) .
$$

Turning to the $(i j)$-component, we find the term with the Riemann tensor contains neither $J=0$ nor 1 mode (see Appendix C). Hence the vanishing of $J=0,1$ modes implies

$$
\left[f_{\alpha(i}(T) f^{\alpha}{ }_{j) k l}(T)\right]_{J=0,1}=O\left(\frac{G m}{L} \frac{1}{L^{2}}\right),
$$

where $[\cdots]_{J=0,1}$ means the $J=0,1$ parts of the quantity.

Let us now turn to the modes higher than $J=1$. The $(T T)$-component contains $J=2$ mode, but it just determines the physical perturbation of the internal metric. The higher modes are absent from the beginning. The 
(Ti)-component contains $J=2$ and 3 modes. As for the $J=2$ mode, it does not involve $f_{i j k}^{\alpha}$. Hence it also gives the physical perturbation of the internal metric. The $J=3$ mode of the $(T i)$-component of $\underset{(0)}{h} a b$ is given by

$$
\frac{1}{2} \frac{d z^{\alpha}}{d T}(T) f_{\alpha<i j k>}(T) X^{j} X^{k}+O\left(\frac{G m}{L} \frac{|X|^{2}}{L^{2}}\right),
$$

where $\langle i j k>$ means to take the symmetric traceless part of the indices (see Appendix C). Since this is a pure gauge degree of freedom, we may set it to vanish. Then combining this with Eq. (4.26), we find

$$
\frac{d z^{\alpha}}{d T}(T) f_{\alpha i j k}(T)=O\left(\frac{G m}{L} \frac{1}{L^{2}}\right) .
$$

We do not discuss the higher $J$ modes in the $(i j)$-component, since it does not give us any information which is necessary to derive the equation of motion.

Then, from Eqs. (4.14), (4.22) and (4.29), we find

$$
\frac{d z^{\alpha}}{d T}(T) \sigma_{; \alpha}(x(T, X), z(T))=-\frac{d z^{\alpha}}{d T}(T) \mathcal{F}_{\alpha}(T, X)=O\left(\frac{|X|^{4}}{L^{4}} L\right),
$$

to the lowest order in $G m / L$. Comparing this with the hypersurface condition of $\tau_{x}$, Eq. (2.23), one finds that the $T=$ constant hypersurface differs from the $\tau_{x}=$ constant hypersurface only by $O\left(\epsilon^{4}\right)=O\left(|X|^{4}\right)$. Then following the calculations done in section 2 again, one finds they are unaltered even if we replace Eq. (2.23) with

$$
\left[\sigma_{; \alpha}(x, z(\tau)) \dot{z}^{\alpha}(\tau)\right]_{\tau=\tau_{x}}=O\left(\epsilon^{4}\right) .
$$

The only effect of this replacement is to add $O\left(\epsilon^{4}\right)$ to the right hand side of the equivalent formula (A13) in Appendix A. Thus $T$ can be identified $\tau_{x}$ to the lowest order in $G m / L$. The reason why we have done the $\left(\begin{array}{l}2 \\ 0\end{array}\right)$ matching prior to the remaining $\left(\begin{array}{l}0 \\ 1\end{array}\right)$ matching is to establish this equivalence of $T$ and $\tau_{x}$.

\section{External Perturbation; $\left(\begin{array}{l}0 \\ 1\end{array}\right)$ Matching}

Now we proceed to the first non-trivial order in $G m /|X|$. For this purpose, we must develop the external scheme. However, since the time slicing by the internal time coordinate $T$ is now identical to that by $\tau_{x}$ in the lowest order in $G m / L$, we can use the previously obtained formula (2.27) for the external metric perturbation.

There remains the $\left(\begin{array}{l}0 \\ 1\end{array}\right)$ matching among the the matchings which becomes of $O\left((G m / L)^{1 / 2}\right)$ in the matching region. This matching relates the masses of the particle in both schemes. Since this matching is of $O\left((|X| / L)^{0}\right)$, it is allowed to consider as if the background external metric were flat. As is well-known, the linear perturbation induced by a pointlike particle of mass $m$ in the flat background spacetime is exactly equal to the asymptotic metric of a Schwarzschild black hole of mass $m$ in the linear order in $m$. This fact indicates that the matching gives a consistency condition at this order.

In order to directly check the consistency, we rewrite Eq. (2.27) in terms of the internal coordinates. From (4.10), we have only to consider the first term of the right hand side of Eq. (2.27). Using Eqs. (4.6), (4.7) and the fact that $\epsilon=\sqrt{\sigma_{; \alpha} \sigma^{; \alpha}}=\sqrt{\mathcal{F}_{\alpha}(T, X) \mathcal{F}^{\alpha}(T, X)}$, the trace-reverse of Eq. (2.27) is transformed to give

$$
G m \underset{(1)}{\stackrel{(0)}{h} a b}(X) d X^{a} d X^{b}=G m\left(\frac{2}{|X|} d T^{2}+\frac{2}{|X|} d X^{i} d X^{i}\right),
$$

which corresponds to the asymptotic form of the Schwarzschild black hole of mass $m$ in the harmonic coordinates.

\section{E. Radiation Reaction; $\left(\begin{array}{l}1 \\ 1\end{array}\right)$ and $\left(\begin{array}{l}2 \\ 1\end{array}\right)$ Matchings}

There are many components which become of $O\left((G m / L)^{1}\right)$ and $O\left((G m / L)^{3 / 2}\right)$ in the matching region. However, we are interested in the leading order correction to the equation of motion with respect to $G m / L$ and we found in subsection B that in the lowest order the terms which behave as $\sim|X|^{0}$ or $|X|^{1}$ determines the motion of the particle. Therefore we consider the $\left(\begin{array}{l}1 \\ 1\end{array}\right)$ and $\left(\begin{array}{l}2 \\ 1\end{array}\right)$ matchings here. 
In order to perform the $\left(\begin{array}{l}1 \\ 1\end{array}\right)$ and $\left(\begin{array}{l}2 \\ 1\end{array}\right)$ matchings, the calculation in obtaining Eq. (4.32) must be done to the linear order in $|X|$. This can be done in the same way with the aid of the expression of $\kappa^{2}(x)$ given in Appendix A. Using further the results obtained in subsections $\mathrm{B}$ and $\mathrm{C}$, we find the following matching equations:

$$
\begin{aligned}
& \frac{G m}{L} \stackrel{(1)}{H}_{(1)} T T=\left\{\left(\frac{d z}{d T}\right)^{2}(T)+1\right\}+G m \frac{d z^{\alpha}}{d T}(T) \frac{d z^{\beta}}{d T}(T) \Theta_{\alpha \beta}(T)+O\left(\left(\frac{G m}{L}\right)^{2}\right), \\
& \text { (TT)-component, } \\
& \frac{G m}{L} \underset{(1)}{\stackrel{(1)}{H}} T i=\frac{d z^{\alpha}}{d T}(T) f_{\alpha i}(T)+G m \frac{d z^{\alpha}}{d T}(T) f^{\beta}{ }_{i}(T) \Theta_{\alpha \beta}(T)+O\left(\left(\frac{G m}{L}\right)^{2}\right), \\
& \text { (Ti)-component, } \\
& \frac{G m}{L} \stackrel{(1)}{H}_{(1)}{ }_{i j}=\left\{f_{i}^{\alpha}(T) f_{\alpha j}(T)-\delta_{i j}\right\}+G m f^{\alpha}{ }_{i}(T) f^{\beta}{ }_{j}(T) \Theta_{\alpha \beta}(T)+O\left(\left(\frac{G m}{L}\right)^{2}\right) \text {, } \\
& \text { (ij)-component, } \\
& \frac{G m}{L^{2}} \stackrel{(2)}{H}_{(1)} T T=2 \frac{d z^{\alpha}}{d T}(T) \frac{D f_{\alpha i}}{d T}(T) X^{i} \\
& +G m\left\{\frac{d z^{\alpha}}{d T}(T) \frac{d z^{\beta}}{d T}(T) f_{i}^{\gamma}(T) \Theta_{\alpha \beta \gamma}(T) X^{i}-\frac{1}{3|X|^{3}} f_{\alpha i}(T) f^{\alpha}{ }_{j k l}(T) X^{i} X^{j} X^{k} X^{l}\right. \\
& \left.-\frac{5}{3|X|} R_{\alpha \beta \gamma \delta}(z(T)) \frac{d z^{\alpha}}{d T}(T) f^{\beta}{ }_{i}(T) \frac{d z^{\gamma}}{d T}(T) f^{\delta}{ }_{j}(T) X^{i} X^{j}\right\}+O\left(\left(\frac{G m}{L}\right)^{2} \frac{|X|}{L}\right), \\
& \text { (TT)-component, } \\
& \frac{G m}{L^{2}} \underset{(1)}{\stackrel{(2)}{H}} T i=\frac{d z^{\alpha}}{d T}(T) f_{\alpha i j}(T) X^{j}+f_{i}^{\alpha}(T) \frac{D f_{\alpha j}}{d T}(T) X^{j} \\
& +G m\left\{\frac{d z^{\alpha}}{d T}(T) f_{i}^{\beta}(T) f_{j}^{\gamma}(T) \Theta_{\alpha \beta \gamma}(T) X^{j}-2 R_{\alpha \beta \gamma \delta}(z(T)) \frac{d z^{\alpha}}{d T}(T) f^{\beta}{ }_{i}(T) \frac{d z^{\gamma}}{d T}(T) f^{\delta}{ }_{j}(T) X^{j}\right. \\
& \left.-\frac{2}{3|X|} R_{\alpha \beta \gamma \delta}(z(T)) \frac{d z^{\alpha}}{d T}(T) f^{\beta}{ }_{j}(T) f^{\gamma}{ }_{i}(T) f^{\delta}{ }_{k}(T) X^{j} X^{k}\right\}+O\left(\left(\frac{G m}{L}\right)^{2} \frac{|X|}{L}\right),
\end{aligned}
$$

(Ti)-component,

where

$$
\begin{aligned}
G m \Theta_{\alpha \beta}(T) & :=h_{(v) \alpha \beta}(z(T)), \\
G m \Theta_{\alpha \beta \gamma}(T) & :=h_{(v) \alpha \beta ; \gamma}(z(T)),
\end{aligned}
$$

with

$$
h_{(v) \mu \nu}(x):=\psi_{(v) \mu \nu}(x)-\frac{1}{2} g_{\mu \nu}(x) \psi_{(v)}(x) .
$$

Note that $h_{(v) \mu \nu}(x)$ is the metric perturbation due to $v_{\mu \nu \alpha \beta}(x, z)$ in the Green function. The $(i j)$-component of the $\left(\begin{array}{l}2 \\ 1\end{array}\right)$ matching is not presented here since it will not be used in the following discussion.

According to the discussion given in subsection B we have $\stackrel{(1)}{H}_{a b}=0$. Thus the right hand sides of Eqs. (4.33), (4.34) (1)

and (4.35) must vanish. As for $\underset{(1)}{\stackrel{(2)}{H}}$ ab , we cannot set them equal to zero. However, again from the condition that there are no $J=0,1$ modes, the corresponding parts in the right hand sides of Eqs. (4.36) and (4.37) must vanish if they are extracted out. Following the discussion given in Appendix C, one finds the terms involving the Riemann tensor contains no $J=0,1$ mode. Furthermore, the $J=0,1$ mode of the term involving $f^{\alpha}{ }_{j k l}$ in Eq. (4.36) is proportional to

$$
\left[f_{\alpha(i}(T) f^{\alpha}{ }_{j) k l}(T)\right]_{J=0,1}
$$


which vanishes at the lowest order in $G m / L$ by Eq. (4.27). Hence the $J=1$ modes of the remaining terms in Eqs. (4.36) and (4.37) are extracted out to give

$$
\begin{aligned}
0= & 2 \frac{d z^{\alpha}}{d T}(T) \frac{D f_{\alpha i}}{d T}(T)+G m \frac{d z^{\alpha}}{d T}(T) \frac{d z^{\beta}}{d T}(T) f_{i}^{\gamma}(T) \Theta_{\alpha \beta \gamma}(T)+O\left(\left(\frac{G m}{L}\right)^{2} \frac{1}{L}\right), \\
& (T T) \text {-component, } \\
0= & f_{\alpha[i}(T) \frac{D f^{\alpha}{ }_{j]}}{d T}(T)+G m \Theta_{\alpha \beta \gamma}(T) \frac{d z^{\alpha}}{d T}(T) f_{[i}^{\beta}(T) f_{j]}^{\gamma}(T)+O\left(\left(\frac{G m}{L}\right)^{2} \frac{1}{L}\right),
\end{aligned}
$$

(Ti)-component.

The $J=0$ mode of Eq. (4.36) is shown to vanish, while that of Eq. (4.37) exists but it does not contain useful information. The matching condition for this mode just gives the equation which determines $\left(d z^{\alpha} / d T\right) f_{\alpha i i}$ to the first order in $G m / L$.

The covariant $T$-derivative of Eq. (4.33) and that of Eq. (4.34) with Eq. (4.41) give the equation of motion with leading correction due to the radiation reaction,

$$
\frac{D}{d T} \frac{d z^{\alpha}}{d T}(T)=-\frac{G m}{2}\left(\Theta^{\alpha}{ }_{\beta \gamma}(T)+\Theta^{\alpha}{ }_{\gamma \beta}(T)-\Theta_{\beta \gamma}{ }^{\alpha}(T)\right) \frac{d z^{\beta}}{d T}(T) \frac{d z^{\gamma}}{d T}(T)+O\left(\left(\frac{G m}{L}\right)^{2} \frac{1}{L}\right) .
$$

The leading correction to the evolution of the 'triad' basis, $f^{\alpha}{ }_{i}(T)$, are also obtained from Eq. (4.41) and the covariant $T$-derivatives of Eq. (4.35) with Eq. (4.42) as

$$
\frac{D}{d T} f_{i}^{\alpha}(T)=-\frac{G m}{2}\left(\Theta_{\beta \gamma}^{\alpha}(T)+\Theta^{\alpha}{ }_{\gamma \beta}(T)-\Theta_{\beta \gamma}{ }^{\alpha}(T)\right) f^{\beta}{ }_{i}(T) \frac{d z^{\gamma}}{d T}(T)+O\left(\left(\frac{G m}{L}\right)^{2} \frac{1}{L}\right) .
$$

Since the internal time coordinate $T$ is not properly normalized in the external metric, we define the proper time, $\tau=\tau(T)$, such that $(d z / d \tau)^{2}=-1$. It is easy to see that we should choose

$$
\frac{d \tau}{d T}=1+\frac{G m}{2} \Theta_{\alpha \beta}(\tau) \frac{d z^{\alpha}}{d \tau}(\tau) \frac{d z^{\beta}}{d \tau}(\tau)+O\left(\left(\frac{G m}{L}\right)^{2}\right) .
$$

Since the second term on the right hand side of this equation is proportional to the small perturbation induced by the particle, it is guaranteed to stay small even after a long time interval compared with the reaction time scale $\tau_{r}=O\left((G m / L)^{-1} L\right)$. Then Eq. (4.43) becomes

$$
\frac{D}{d \tau} \frac{d z^{\alpha}}{d \tau}(\tau)=-\frac{G m}{2}\left(\frac{d z^{\alpha}}{d \tau} \frac{d z^{\beta}}{d \tau} \frac{d z^{\gamma}}{d \tau} \frac{d z^{\delta}}{d \tau}+2 g^{\alpha \beta}(z) \frac{d z^{\gamma}}{d \tau} \frac{d z^{\delta}}{d \tau}-g^{\alpha \delta}(z) \frac{d z^{\beta}}{d \tau} \frac{d z^{\gamma}}{d \tau}\right)(\tau) \Theta_{\beta \gamma \delta}(\tau)+O\left(\left(\frac{G m}{L}\right)^{2} \frac{1}{L}\right) .
$$

It is easy to see that this equation is identical to that obtained at the end of the previous section.

Also, the triad basis are not properly normalized in the external metric. Thus we define, $e^{\alpha}{ }_{i}(\tau)$, such that $e_{\alpha i}(\tau) e^{\alpha}{ }_{j}(\tau)=\delta_{i j}$ and $e^{\alpha}{ }_{i}(\tau)=\left(\delta_{i j}+s_{i j}\right) f^{\alpha}{ }_{j}-G m\left(d z^{\alpha} / d T\right)\left(d z^{\beta} / d T\right) f^{\gamma}{ }_{i} \Theta_{\beta \gamma}$, where $s_{i j}$ is of $O(G m / L)$ and the last term is added so as to satisfy the orthonormal condition, $e_{\alpha i}(\tau)\left(d z^{\alpha} / d \tau\right)(\tau)=0$ (see Eq. (4.34)). We find

$$
s_{i j}=-\frac{G m}{2} \Theta_{\alpha \beta}(\tau) f^{\alpha}{ }_{i}(\tau) f^{\beta}{ }_{j}(\tau)+O\left(\left(\frac{G m}{L}\right)^{2}\right),
$$

and again this is guaranteed to stay small. Then the evolution of the normalized triad $e^{\alpha}{ }_{i}(\tau)$ becomes

$$
\frac{D}{d \tau} e^{\alpha}{ }_{i}(\tau)=-\frac{G m}{2}\left(\frac{d z^{\alpha}}{d \tau} \frac{d z^{\beta}}{d \tau} e^{\gamma}{ }_{i} \frac{d z^{\delta}}{d \tau}+g^{\alpha \beta}(z) \frac{d z^{\gamma}}{d \tau} e^{\delta}{ }_{i}-g^{\alpha \delta}(z) e^{\beta}{ }_{i} \frac{d z^{\gamma}}{d \tau}\right)(\tau) \Theta_{\beta \gamma \delta}(\tau)+O\left(\left(\frac{G m}{L}\right)^{2} \frac{1}{L}\right) .
$$




\section{IMPLICATIONS}

In this section, we first consider the physical meaning of the equation of motion obtained in the preceding two sections. Since the equation of motion we have obtained contains an unknown function $v_{\mu \nu \alpha \beta}(x, z)$, we need to give a method to explicitly determine the particle trajectory. Here we consider a couple of possibilities to calculate it in the case of a specific background, such as Kerr spacetime.

In order to make the meaning of the equation of motion manifest, we divide the perturbed metric in the external scheme into two pieces as

$$
h_{\mu \nu}(x)=h_{(u) \mu \nu}(x)+h_{(v) \mu \nu}(x)
$$

where $h_{(v) \mu \nu}(x)$ is the part due to the tail term (defined in Eq. 4.39)) and $h_{(u) \mu \nu}(x)$ is the part due to the $u_{\mu \nu \alpha \beta}$ term in the Green function (corresponding to the first term on the right hand side of Eq. (2.20). The singular behavior of the perturbed metric in the coincidence limit is totally due to $h_{(u) \mu \nu}(x)$. Thus, we introduce the regularized perturbed spacetime defined by

$$
\tilde{g}_{(v) \mu \nu}(x):=g_{\mu \nu}(x)+h_{(v) \mu \nu}(x),
$$

which has no singular behavior any more. Then we find the equation of motion $(4.43)$ and the evolution equation of the triad basis (4.44) coincide with the geodesic equation and the geodetic parallel transport, respectively, in this regularized perturbed spacetime, $\tilde{g}_{(v) \mu \nu}$. To see this let us consider the parallel transport of a vector $A^{\alpha}$ along a geodesic $x^{\alpha}=z^{\alpha}(\tilde{\tau})$ in this spacetime. It is given by

$$
\frac{\tilde{D}_{(v)}}{d \tilde{\tau}} A^{\alpha}:=\frac{D}{d \tilde{\tau}} A^{\alpha}+\delta \Gamma_{(v)}{ }^{\alpha}{ }_{\beta \gamma} A^{\beta} \frac{d z^{\gamma}}{d \tilde{\tau}}=0
$$

to the linear order in $h_{(v) \nu \mu}$ where

$$
\delta \Gamma_{(v)}^{\alpha}{ }_{\beta \gamma}:=\frac{1}{2}\left(h_{(v)}^{\alpha}{ }_{\beta ; \gamma}+h_{(v)}^{\alpha}{ }_{\gamma ; \beta}-h_{(v) \beta \gamma} ; \alpha\right) .
$$

Then one recovers Eqs. (4.43) and (4.44) by identifying $\tilde{\tau}$ with $T$ and replacing $A^{\alpha}$ with $d z^{\alpha} / d T$ or $f^{\alpha}{ }_{i}$. This fact is the main result of this paper.

We note that the present equation of motion is analogous to that in the electromagnetic case, except that the instantaneous reaction force which is proportional to higher derivatives of the particle velocity is absent in the present case. This is because the particle traces the geodesic in the lowest order of approximation. If an external force field exists, the assumption of the geodesic motion in the lowest order breaks down and furthermore the contribution of the external force field to the energy momentum tensor must be taken into account. Since this fact makes the problem too complicated, it is beyond the scope of the present paper.

Now let us consider the way how to construct $\tilde{g}_{(v) \mu \nu}$. As stated in the beginning of this section, in order to evaluate the particle trajectory explicitly, a practical scheme to calculate $\tilde{g}_{(v) \mu \nu}$ must be developed. Unfortunately, we do not have any scheme which can be satisfactorily applied when calculating the particle trajectory with the effect of the radiation reaction, even on a specific background spacetime such as a Kerr black hole. Here we just give a few primitive discussions on this matter. For definiteness, we focus on the case of the retarded boundary condition.

Basically, there seems to be two approaches for calculating $\tilde{g}_{(v) \mu \nu}$ (or equivalently $h_{(v) \mu \nu}$ ). The first one is to calculate $h_{(v) \mu \nu}$ directly. The second one is to calculate $h_{\mu \nu}=h_{(u) \mu \nu}+h_{(v) \mu \nu}$ and subtract $h_{(u) \mu \nu}$ from it. In the following, we discuss only the first approach. As for the second approach, we have nothing to mention here, but this direction of research may be fruitful.

By definition, $h_{(u) \mu \nu}$ evaluated on the particle trajectory is independent of the past history of the particle.** Therefore if we consider the metric defined by

$$
h_{\mu \nu}^{(\Delta \tau)}(x)=\left(\delta_{\mu}^{\rho} \delta_{\nu}^{\sigma}-\frac{1}{2} g_{\mu \nu}(x) g^{\rho \sigma}(x)\right) \int_{-\infty}^{\tau_{x}-\Delta \tau} d \tau^{\prime} G_{\rho \sigma \alpha \beta}^{R e t}\left(x, z\left(\tau^{\prime}\right)\right) \dot{z}^{\alpha}\left(\tau^{\prime}\right) \dot{z}^{\beta}\left(\tau^{\prime}\right),
$$

for any finite $\Delta \tau(>0)$, it will not contain $h_{(u) \mu \nu}$ when it is evaluated on the particle trajectory. The difference between $h_{\mu \nu}^{(\Delta \tau)}$ and $h_{(v) \mu \nu}$ comes from the integral over the small interval,

\footnotetext{
** There is a possibility that the future light cone emanating from $z$ crosses the particle trajectory. Since inclusion of this possibility makes the problem too complicated, we do not consider it here.
} 


$$
\sim G m \int_{\tau_{x}-\Delta \tau}^{\tau_{x}} d \tau^{\prime} v_{\rho \sigma \alpha \beta}\left(x, z\left(\tau^{\prime}\right)\right) \dot{z}^{\alpha}\left(\tau^{\prime}\right) \dot{z}^{\beta}\left(\tau^{\prime}\right)
$$

Since $v_{\rho \sigma \alpha \beta}(x, z)$ is a regular function, this integral will be negligible for a sufficiently small $\Delta \tau$. Thus $\lim _{\Delta \tau \rightarrow 0} h_{\mu \nu}^{(\Delta \tau)}$ will give $h_{(v) \mu \nu}$.

In the case of the electromagnetic (vector) Green function, a calculation along the above strategy was performed by DeWitt and DeWitt [9] by assuming the background gravitational field is weak so that its metric is given by the small perturbation on the Minkowski metric,

$$
g_{\mu \nu}=\eta_{\mu \nu}+h_{\mu \nu}^{(b)}
$$

DeWitt and DeWitt calculated the relevant part of the Green function perturbatively to the first order in $h_{\mu \nu}^{(b)}$ by using the Minkowski Green function. An analogous calculation seems possible in the case of gravity to evaluate $h_{(v) \mu \nu}$, though it seems difficult to develop such calculations to higher orders in $h_{\mu \nu}^{(b)}$.

As an alternative approach, we give below a speculation of a new method in which we do not have to assume the deviation from the Minkowski spacetime to be small. The standard perturbation formalism of a Kerr black hole gives us the retarded Green function schematically in the form, 周

$$
G_{\mu \nu \alpha \beta}^{R e t}(x, z) \sim \sum_{j, m} \int d \omega \frac{1}{W_{j, m, \omega}} e^{-i \omega\left(t_{x}-t_{z}\right)} h_{\mu \nu}^{j, m, \omega}\left(r_{x}, \theta_{x}, \varphi_{x}\right) h_{\alpha \beta}^{j, m, \omega}\left(r_{z}, \theta_{z}, \varphi_{z}\right),
$$

where $h_{\mu \nu}^{j, m, \omega}(r, \theta, \varphi)$ represents the spatial mode functions with appropriate boundary conditions and $W_{j, m, \omega}$ is the Wronskian. Here we stress the following point. Provided that $x$ is inside the future light cone emanating from $z$, the integrand becomes exponentially small as the imaginary part of $\omega$ goes to $-\infty$. Thus the contour of the $\omega$ integration can be closed in the lower half complex plane of $\omega$. Since the integrand has poles at the quasi-normal mode frequencies, at which $W_{j, m, \omega}$ vanishes, the $\omega$-integral can be transformed into the summation over the contributions from the residues of the poles. We denote this expression of the retarded Green function by

$$
G_{\mu \nu \alpha \beta}^{\operatorname{Ret}(Q N)}(x, z) \sim-2 \pi i \sum_{j, m, n}\left(\frac{d W_{j, m, \omega}}{d \omega}\right)_{\omega=\omega_{n}}^{-1} e^{-i \omega_{n}\left(t_{x}-t_{z}\right)} h_{\mu \nu}^{j, m, \omega_{n}}\left(r_{x}, \theta_{x}, \varphi_{x}\right) h_{\alpha \beta}^{j, m, \omega_{n}}\left(r_{z}, \theta_{z}, \varphi_{z}\right),
$$

where $\omega_{n}$ are the quasi-normal mode frequencies. On the other hand, when $x$ coincides with $z$, the replacement of the $\omega$-integration into the summation over the quasi-normal modes is not allowed. Therefore $\lim _{x \rightarrow z} G_{\mu \nu \alpha \beta}^{\operatorname{Ret}(Q N)}(x, z)$ is not equal to $\lim _{x \rightarrow z} G_{\mu \nu \alpha \beta}^{\operatorname{Ret}}(x, z)$. In particular, we expect that $G_{\mu \nu \alpha \beta}^{\operatorname{Ret}(Q N)}(x, z)$ is regular in the coincidence limit $x \rightarrow z$, since $\left(d W_{j, m, \omega} / d \omega\right)_{\omega=\omega_{n}}$ is known to behave as $1 / n$ ! 10]. Thus it may give the regularized retarded Green function which we want to obtain.

Before closing this section, we give comments on some proposals to the equation of motion including the effect of the gravitational radiation reaction. One is the use of the radiative Green function (a half of the difference between the retarded and advanced Green functions) in the case of a Kerr background [11 As seen easily by using the results obtained in the previous section, the use of the radiative Green function instead of the retarded one results in the replacement of $\psi_{(v) \mu \nu}(x)$ by $\psi_{(v) \mu \nu}^{R a d}(x)$, which is defined by

$$
\psi_{(v)}^{R a d}{ }_{\beta \gamma}(x):=-G m \int_{-\infty}^{+\infty} d \tau^{\prime} v_{\beta \gamma \alpha^{\prime} \beta^{\prime}}\left(x, z\left(\tau^{\prime}\right)\right) \dot{z}^{\alpha^{\prime}}\left(\tau^{\prime}\right) \dot{z}^{\beta^{\prime}}\left(\tau^{\prime}\right)
$$

Gal'tsov has proved that the back reaction force computed using the radiative Green function correctly gives the loss of the energy and the z-component of the angular momentum of the particle in quasi-periodic orbits. However, we do not think that this fact indicates the correctness of the prescription because those constants of motion are special ones which reflect the existence of the corresponding Killing vector field. For example, it is still uncertain if the radiative Green function is useful in evaluating radiation reaction effect on the Carter constant. We would like to come back to this point in future publication.

Recently Ori has argued also in the case of a Kerr background that the partial wave decomposition of the retarded Green function with respect to the spheroidal harmonics as schematically shown above would result in the automatic removal of the divergence when averaged over several orbital periods [12], thus making it possible to derive the

\footnotetext{
${ }^{\dagger \dagger}$ Note that this expression is only schematic, just for the present explanation. The correct expression can be fond in $[1]$.
} 
radiation reaction to the Carter constant. We do not think his argument works. Let us consider expansion of the Newtonian potential of a particle off the origin of the coordinates by using the spherical harmonics. If one calculate the self-force on the particle, one finds the contribution from each partial wave is finite. However, the net self-force is intrinsically ill-defined and if one sums up all the contributions from the different modes, it is easy to see that thus obtained self force diverges. Thus the finiteness of the partial wave contributions does not imply that of their sum.

\section{CONCLUSION}

In this paper, we have derived the equation of motion of a particle on a given background including the effect of the gravitational radiation reaction, i.e., with corrections of order $(G m)$ where $m$ is the mass of the particle. Although we use the terminology 'radiation reaction' here, it may not be appropriate in a strict sense because the equation of motion we have derived may well contain something more than just the radiation reaction. In fact, in the electromagnetic case, the existence of the effect which can be termed as 'the induced polarization force on the background spacetime' is reported by many authors [13]. The existence and the physical meaning of it in the gravitational case is left for future study.

We have derived the equation of motion in two different ways. First we have considered an extension of the electromagnetic counter part developed by DeWitt and Brehme [5]. Due to the nature of gravitational interaction, there appears an ambiguity concerning the renormalization of mass of the particle, which cannot be resolved within this approach. We have then derived the equation of motion by setting an ansatz which seems physically reasonable without justification. In order to overcome this problem, we have developed a method based on the matched asymptotic expansion, assuming the local geometry around the particle is described by a spherically symmetric black hole plus tidal perturbations. This latter approach has proved to be very powerful and we have succeeded in obtaining the same equation of motion as obtained in the former approach.

The correction term of $O(G m)$ is found to be entirely given by the part of the metric perturbation which is due to the tail term of the Green function. Defining the regularized metric as the background plus this tail part of the perturbation, we have found that the equation of motion is the geodesic equation on this regularized perturbed metric.

It is important to note that the particle does not have to be a black hole but the resultant equation of motion can be equally applicable to any compact bodies such as neutron stars. The essential assumption here is that the only scale associated with the particle is $G m$ and the structure is basically spherically symmetric. In this sense, we have shown the strong equivalence principle to the first order in $\mathrm{Gm}$.

We also emphasize that our result gives a justification of the so-called black hole perturbation approach for the first time. In the black hole perturbation approach, one calculates the gravitational radiation from a particle orbiting a black hole with the assumption that the particle is a point-like object with the energy momentum tensor described by the delta function. Although this approach has been fruitful, there has been always skepticism about the validity of the delta functional source. What we have shown in this paper justifies the use of the delta function in the source energy momentum tensor.

In this paper, we has considered a particle which is essentially structureless. A next step will be to include the intrinsic angular momentum of the particle, i.e, to consider a spinning particle. As we have found the method of the matched asymptotic expansion is very powerful, there is a big possibility that we can extend the present analysis to the case of a spinning particle. Extension in this direction is under study.

\section{ACKNOWLEDGMENTS}

We thank T. Futamase, T. Nakamura, M. Shibata, H. Tagoshi, K.S. Thorne and A.G. Wiseman for fruitful conversations. Y. M. thanks Prof. H. Sato and Prof. S. Ikeuchi for their continuous encouragements. This work was supported in part by Monbusho Grant-in-Aid for Scientific Research No. 5427.

\section{APPENDIX A: NOTATION AND BASIC FORMULAS}

Here in this appendix, we summarize our notation and basic formulas. As for the formulas already derived in DB, we just write down the results.

\section{Basic Notation}

(i) The Riemann tensor and the Ricci tensor are defined by

$$
\begin{aligned}
R_{\nu \xi \rho}^{\mu} & :=\Gamma_{\nu \rho, \xi}^{\mu}-\Gamma_{\nu \xi, \rho}^{\mu}+\Gamma_{\sigma \xi}^{\mu} \Gamma_{\nu \rho}^{\sigma}-\Gamma_{\sigma \rho}^{\mu} \Gamma_{\nu \xi}^{\sigma} \\
R_{\mu \nu} & :=R_{\mu \xi \nu}^{\xi} \\
R & :=R_{\mu}^{\mu} .
\end{aligned}
$$


(ii) Symmetrization and anti-symmetrization of the tensor indices are described by $t^{(\mu \nu)}=\left(t^{\mu \nu}+t^{\nu \mu}\right) / 2$ and $t^{[\mu \nu]}=$ $\left(t^{\mu \nu}-t^{\nu \mu}\right) / 2$, respectively.

(iii) For an arbitrary bi-tensor $Q$,

$$
\begin{aligned}
& Q_{; \alpha}\left(x, z\left(\tau_{x}\right)\right):=\left[Q_{; \alpha}(x, z)\right]_{z=z\left(\tau_{x}\right)}, \\
& Q_{; \mu}\left(x, z\left(\tau_{x}\right)\right):=\left[Q_{; \mu}(x, z)\right]_{z=z\left(\tau_{x}\right)},
\end{aligned}
$$

while

$$
\left[Q\left(x, z\left(\tau_{x}\right)\right)\right]_{; \mu}:=Q_{; \mu}\left(x, z\left(\tau_{x}\right)\right)+Q_{; \alpha}\left(x, z\left(\tau_{x}\right)\right) \dot{z}^{\alpha}\left(\tau_{x}\right) \tau_{x ; \mu} .
$$

(iv) The basic equations satisfied by a half the squared geodetic interval $\sigma(x, z)$ (Eqs. (1.11) and (1.12) of DB):

$$
\begin{aligned}
& \sigma(x, z)=\frac{1}{2} g^{\mu \nu}(x) \sigma_{; \mu}(x, z) \sigma_{; \nu}(x, z)=\frac{1}{2} g^{\alpha \beta}(z) \sigma_{; \alpha}(x, z) \sigma_{; \beta}(x, z), \\
& \lim _{x \rightarrow z} \sigma(x, z)=0 .
\end{aligned}
$$

(v) The defining equations of the parallel displacement bi-vector (Eqs. (1.31) and (1.32) of DB):

$$
\begin{aligned}
& \bar{g}_{\mu \alpha ; \nu}(x, z) g^{\nu \sigma}(x) \sigma_{; \sigma}(x, z)=0, \quad \bar{g}_{\mu \alpha ; \beta}(x, z) g^{\beta \gamma}(z) \sigma_{; \gamma}(x, z)=0, \\
& \lim _{x \rightarrow z} \bar{g}_{\mu}{ }^{\alpha}(x, z)=\delta_{\mu}{ }^{\alpha} .
\end{aligned}
$$

(vi) The definition of $\Delta(x, z)$ (Eqs. (1.50), (1.51), (1.60) and (1.61) of DB):

$$
\Delta(x, z):=\left|\bar{g}^{\alpha \mu}(z, x) \sigma_{; \mu \beta}(x, z)\right| .
$$

\section{Basic formulas}

(i) (Eq. (1.28) of DB)

$$
\sigma_{; \alpha \beta}(x, z)=g_{\alpha \beta}(z)+\frac{1}{3} R_{\alpha}{ }^{\delta}{ }^{\delta}(z) \sigma_{; \gamma}(x, z) \sigma_{; \delta}(x, z)+O\left(\epsilon^{3}\right) .
$$

(ii)

$$
\begin{aligned}
\bar{g}_{; \beta}^{\mu \alpha}(x, z) & =\frac{1}{2} \bar{g}^{\mu \gamma}(x, z) R_{\gamma \beta \delta}^{\alpha}(z) \sigma^{; \delta}(x, z)+O\left(\epsilon^{2}\right), \\
\bar{g}_{; \nu}^{\mu \alpha}(x, z) & =\frac{1}{2} \bar{g}^{\mu \beta}(x, z) \bar{g}_{\nu}{ }^{\gamma}(x, z) R_{\beta \gamma \delta}^{\alpha}(z) \sigma^{; \delta}(x, z)+O\left(\epsilon^{2}\right),
\end{aligned}
$$

which can be obtained from Eqs. (1.40) and (1.41) of DB.

(iii) (Eqs. (1.51), (1.64) and (1.73) of DB)

$$
\sigma_{; \mu \beta}(x, z)=-\bar{g}_{\mu}{ }^{\alpha}(x, z)\left(g_{\alpha \beta}(z)-\frac{1}{6} R_{\alpha \gamma \beta \delta}(z) \sigma^{; \gamma}(x, z) \sigma^{; \delta}(x, z)\right)+O\left(\epsilon^{3}\right) .
$$

(iv) Here we give the formulas which we need to expand Eq. (2.20) to obtain Eq. (2.27). Since we want to express Eq. (2.20) in terms of $\tau_{x}$ defined by Eq. (2.23) instead of $\tau_{\text {Ret/Adv }}(x)$, we expand each factor of each term which consists of Eq. (2.20) with $\delta_{\text {Ret/Adv }}(x)$, (2.26). We first consider $[\dot{\sigma}(x, z(\tau))]_{\tau=\tau_{\text {Ret } / A d v}(x)}$, which is expanded as

$$
\begin{aligned}
{[\dot{\sigma}(x, z(\tau))]_{\tau=\tau_{\text {Ret } / A d v}(x)}=\dot{\sigma}(x,} & \left.z\left(\tau_{x}\right)\right)+\ddot{\sigma}\left(x, z\left(\tau_{x}\right)\right) \delta_{\text {Ret } / A d v}(x) \\
& +\frac{1}{2} \dddot{\sigma}\left(x, z\left(\tau_{x}\right)\right) \delta_{\text {Ret } / A d v}^{2}(x)+\frac{1}{3 !} \dddot{\sigma}\left(x, z\left(\tau_{x}\right)\right) \delta_{\text {Ret/Adv }}^{3}(x)+O\left(\epsilon^{4}\right) .
\end{aligned}
$$

Each term is computed as follows; 


$$
\begin{aligned}
\dot{\sigma}\left(x, z\left(\tau_{x}\right)\right)= & \sigma_{; \alpha}\left(x, z\left(\tau_{x}\right)\right) \dot{z}^{\alpha}\left(\tau_{x}\right)=0, \\
\ddot{\sigma}\left(x, z\left(\tau_{x}\right)\right)= & -\kappa^{2}(x) \\
= & \sigma_{; \alpha \beta}\left(x, z\left(\tau_{x}\right)\right) \dot{z}^{\alpha}\left(\tau_{x}\right) \dot{z}^{\beta}\left(\tau_{x}\right)+\sigma_{; \alpha}\left(x, z\left(\tau_{x}\right)\right) \ddot{z}^{\alpha}\left(\tau_{x}\right) \\
= & \left(g_{\alpha \beta}\left(z\left(\tau_{x}\right)\right)+\frac{1}{3} R_{\alpha}^{\gamma} \beta^{\delta}\left(z\left(\tau_{x}\right)\right) \sigma_{; \gamma}\left(x, z\left(\tau_{x}\right)\right) \sigma_{; \delta}\left(x, z\left(\tau_{x}\right)\right)\right) \dot{z}^{\alpha}\left(\tau_{x}\right) \dot{z}^{\beta}\left(\tau_{x}\right) \\
& \quad+\sigma_{; \alpha}\left(x, z\left(\tau_{x}\right)\right) \ddot{z}^{\alpha}\left(\tau_{x}\right)+O\left(\epsilon^{3}\right), \\
\dddot{\sigma}\left(x, z\left(\tau_{x}\right)\right)= & \sigma_{; \alpha}\left(x, z\left(\tau_{x}\right)\right) \dddot{z}^{\alpha}\left(\tau_{x}\right)+O\left(\epsilon^{2}\right), \\
\dddot{\sigma}\left(x, z\left(\tau_{x}\right)\right)= & -g_{\alpha \beta}\left(z\left(\tau_{x}\right)\right) \ddot{z}^{\alpha}\left(\tau_{x}\right) \ddot{z}^{\beta}\left(\tau_{x}\right)+O(\epsilon) .
\end{aligned}
$$

In the above computation, we used Eqs. (A9), (2.23), and the normalization condition $(d z / d \tau)^{2}=-1+O(G m / L)$, which was proved to be consistent in sections 3 and 4 . Then we obtain

$$
\left[\frac{1}{\dot{\sigma}(x, z(\tau))}\right]_{\tau=\tau_{\text {Ret } / \text { Adv }}(x)}= \pm \frac{1}{\epsilon(x) \kappa(x)}\left(1 \mp \frac{1}{3} \epsilon(x) \dddot{z}^{\alpha}\left(\tau_{x}\right) \sigma_{; \alpha}\left(x, z\left(\tau_{x}\right)\right)-\frac{1}{8} \epsilon^{2}(x) \ddot{z}^{2}\left(\tau_{x}\right)+O\left(\epsilon^{3}\right)\right) .
$$

Noting the explicit form of $u^{\mu \nu \alpha \beta}(x, z)$ in Eq. 2.10), it is necessary to compute $\left[\Delta^{1 / 2}(x, z(\tau))\right]_{\tau=\tau_{\text {Ret/Adv }}(x)}$, $\left[\bar{g}_{\mu \alpha}(x, z(\tau))\right]_{\tau=\tau_{\text {Ret/Adv }}(x)}$ and $\left[\dot{z}^{\alpha}(\tau)\right]_{\tau=\tau_{\text {Ret/Adv }}(x)}$. In the same way,

$$
\begin{aligned}
& {\left[\Delta^{1 / 2}(x, z(\tau))\right]_{\tau=\tau_{\text {Ret/Adv }}(x)}=1+O\left(\epsilon^{3}\right),} \\
& {\left[\bar{g}_{\mu \alpha}(x, z(\tau))\right]_{\tau=\tau_{\text {Ret } / A d v}(x)}} \\
& \quad=\bar{g}_{\mu \alpha}\left(x, z\left(\tau_{x}\right)\right) \pm \frac{1}{2} \bar{g}_{\mu}^{\beta}\left(x, z\left(\tau_{x}\right)\right) R_{\alpha \beta \gamma \delta}\left(z\left(\tau_{x}\right)\right) \sigma^{; \gamma}\left(x, z\left(\tau_{x}\right)\right) \dot{z}^{\delta}\left(\tau_{x}\right) \epsilon(x)+O\left(\epsilon^{3}\right), \\
& {\left[\dot{z}^{\alpha}(\tau)\right]_{\tau=\tau_{\text {Ret } / A d v}(x)}=\dot{z}^{\alpha}\left(\tau_{x}\right) \mp \epsilon(x) \kappa^{-1}(x) \ddot{z}^{\alpha}\left(\tau_{x}\right)+\frac{1}{2} \epsilon^{2}(x) \dddot{z} \alpha\left(\tau_{x}\right)+O\left(\epsilon^{3}\right),}
\end{aligned}
$$

Putting them into the first term in the parentheses of Eq. (2.20), and using Eq. (2.11) and the fact

$$
A^{\mu}(x)=\bar{g}_{\alpha}^{\mu}(x, z)\left(A^{\alpha}(z)-\sigma_{; \beta}(x, z) A^{\alpha ; \beta}(z)+O\left(\epsilon^{2}\right)\right),
$$

in computing the second term, we obtain Eq. (2.27).

\section{APPENDIX B: 2ND ORDER VARIATION OF THE EINSTEIN TENSOR}

We derive Eq. (3.8) by taking the variation of the Einstein-Hilbert action. We first compute the Einstein-Hilbert action of the metric, $\tilde{g}_{\mu \nu}=g_{\mu \nu}+h_{\mu \nu}$. For this purpose we define a differential operator $\delta_{\mathbf{g}}$ :

$$
\begin{aligned}
& \delta_{\mathbf{g}} Q(g)=\lim _{\epsilon \rightarrow 0} \frac{Q(g+\epsilon h)-Q(g)}{\epsilon}, \\
& \delta_{\mathbf{g}}^{2} Q(g)=\lim _{\epsilon \rightarrow 0} \frac{Q(g+\epsilon h)+Q(g-\epsilon h)-2 Q(g)}{2 \epsilon^{2}} .
\end{aligned}
$$

We first note the 1st variation of the Einstein tensor taken from a standard text book,

$$
\begin{aligned}
G^{(1) \mu \nu} & =\left(-h^{\mu \nu ; \xi}+h^{\xi \mu ; \nu}+h^{\xi \nu ; \mu}\right)_{; \xi}-h^{; \mu \nu}-g^{\mu \nu}\left(h^{\xi \rho}{ }_{; \xi \rho}-h_{; \xi}^{; \xi}\right) \\
& =\left(-\psi^{\mu \nu ; \xi}+\psi^{\xi \mu ; \nu}+\psi^{\xi \nu ; \mu}\right)_{; \xi}-g^{\mu \nu} \psi_{; \xi \rho}^{\xi \rho},
\end{aligned}
$$

where $\psi_{\mu \nu}=h_{\mu \nu}-(1 / 2) g_{\mu \nu} h$.

Since we are interested only in the 2 nd order variation, we compute the terms proportional to $\mathbf{h}^{2}$ in the action: 


$$
\begin{aligned}
\delta_{\mathbf{g}}^{2}(\sqrt{-g} R)= & \left(\delta_{\mathbf{g}}^{2} \sqrt{-g}\right) R+\left(\delta_{\mathbf{g}} \sqrt{-g} \delta_{\mathbf{g}} g^{\mu \nu}++\sqrt{-g} \delta_{\mathbf{g}}^{2} g^{\mu \nu}\right) R_{\mu \nu} \\
& +\left(\sqrt{-g} \delta_{\mathbf{g}} g^{\mu \nu}+g^{\mu \nu} \delta_{\mathbf{g}} \sqrt{-g}\right) \delta_{\mathbf{g}} R_{\mu \nu}+\sqrt{-g} g^{\mu \nu} \delta_{\mathbf{g}}^{2} R_{\mu \nu} \\
= & -\sqrt{-g}\left(h^{\mu \nu}-\frac{1}{2} g^{\mu \nu} h\right) \delta_{\mathbf{g}} R_{\mu \nu}+\sqrt{-g} g^{\mu \nu} \delta_{\mathbf{g}}^{2} R_{\mu \nu}
\end{aligned}
$$

where we have used the assumption that the background spacetime is vacuum, $R_{\mu \nu}=0$. Now

$$
\begin{aligned}
\delta_{\mathbf{g}} R_{\mu \nu} & =\left(\delta_{\mathbf{g}} \Gamma^{\xi}{ }_{\mu \nu}\right)_{; \xi}-\left(\delta_{\mathbf{g}} \Gamma^{\xi}{ }_{\mu \xi}\right)_{; \nu}, \\
\delta_{\mathbf{g}}^{2} R_{\mu \nu} & =\left(\delta_{\mathbf{g}}^{2} \Gamma^{\xi}{ }_{\mu \nu}\right)_{; \xi}-\left(\delta_{\mathbf{g}}^{2} \Gamma^{\xi}{ }_{\mu \xi}\right)_{; \nu}+\delta_{\mathbf{g}} \Gamma_{\rho \xi}^{\xi} \delta_{\mathbf{g}} \Gamma_{\mu \nu}^{\rho}-\delta_{\mathbf{g}} \Gamma^{\xi}{ }_{\rho \nu} \delta_{\mathbf{g}} \Gamma^{\rho}{ }_{\mu \xi} .
\end{aligned}
$$

Inserting these into Eq. (B5) and using

$$
\delta_{\mathbf{g}} \Gamma_{\mu \nu}^{\xi}=\frac{1}{2}\left(h_{\mu ; \nu}^{\xi}+h_{\nu ; \mu}^{\xi}-h_{\mu \nu} ; \xi\right),
$$

we obtain the 2nd order variation of the Einstein-Hilbert action,

$$
\begin{aligned}
L^{(2)}=\frac{1}{16 \pi G} \frac{1}{\sqrt{-g}} \delta_{\mathbf{g}}^{2}(\sqrt{-g} R) \\
\quad=\frac{1}{64 \pi G}\left[-h_{\mu \nu ; \xi} h^{\mu \nu ; \xi}+2 h_{\mu \nu ; \xi} h^{\xi \mu ; \nu}-2 h_{\mu \nu} ; \nu h^{; \mu}+h_{; \mu} h^{; \mu}\right] \\
\quad=\frac{1}{64 \pi G}\left[-\psi_{\mu \nu ; \xi} \psi^{\mu \nu ; \xi}+2 \psi_{\mu \nu ; \xi} \psi^{\xi \mu ; \nu}+\frac{1}{2} \psi_{; \mu} \psi^{; \mu}\right],
\end{aligned}
$$

where $\psi=\psi^{\alpha}{ }_{\alpha}=-h$ and we have discarded unimportant total divergence terms.

We note

$$
\frac{\delta S}{\delta \tilde{g}_{\mu \nu}}[\tilde{\mathbf{g}}]=\frac{\delta S}{\delta g_{\mu \nu}}[\mathbf{g}+\delta \mathbf{g}]
$$

Thus the 2nd order variation of the Einstein tensor can be obtained by taking the variation of the action with respect to $g_{\mu \nu}$ :

$$
\frac{\delta}{\delta g_{\mu \nu}} \int L^{(2)} \sqrt{-g} d^{4} x=\frac{1}{16 \pi G} \int d^{4} x \sqrt{-g}\left(-G^{(2) \mu \nu}\right)
$$

Hence,

$$
G^{(2) \mu \nu}=-8 \pi G\left[2 \frac{\delta L^{(2)}}{\delta g_{\mu \nu}}+g^{\mu \nu} L^{(2)}\right]
$$

Carrying out the variation of $L^{(2)}$ one finds

$$
\begin{aligned}
G^{(2) \mu \nu}=-\frac{1}{2}[ & \frac{1}{2}\left(\psi^{\mu \xi ; \rho}+\psi^{\mu \rho ; \xi}-\psi^{\xi \rho ; \mu}\right)\left(\psi_{\xi ; \rho}^{\nu}+\psi_{\rho ; \xi}^{\nu}-\psi_{\xi \rho}^{; \nu}\right) \\
& +\psi_{; \rho \xi}^{\mu \xi} \psi^{\rho \nu}+\psi_{; \rho \xi}^{\nu \xi} \psi^{\rho \mu}-\psi_{; \xi \rho}^{\mu \nu} \psi^{\xi \rho}-\left(\psi^{\mu \nu} \psi_{; \rho}^{\xi \rho}\right)_{; \xi}-\frac{1}{4} \psi^{; \mu} \psi^{; \nu} \\
& \left.+\frac{1}{4} g^{\mu \nu}\left[-\psi_{\rho \sigma ; \xi} \psi^{\rho \sigma ; \xi}+2 \psi_{\rho \sigma ; \xi} \psi^{\xi \rho ; \sigma}+\frac{1}{2} \psi_{; \xi} \psi^{; \xi}\right]\right] \\
& +\frac{1}{2} \psi G^{(1) \mu \nu} .
\end{aligned}
$$

Note that if $\mathbf{h}$ is a linear perturbation which satisfies $G^{(1) \mu \nu}[\mathbf{h}]=0$, the tensor $T_{G}^{\mu \nu}:=-(1 / 8 \pi G) G^{(2) \mu \nu}$ describes the conserved energy momentum tensor of the perturbed gravitational field. 


\section{APPENDIX C: TENSOR HARMONICS EXPANSION}

Here we briefly review the construction of the scalar and the vector harmonics in terms of the symmetric trace-free (STF) tensor [14]. We introduce the notation

$$
A_{<i_{1} i_{2} \cdots i_{\ell}>}
$$

to represent the totally symmetric and trace-free part of $A_{i_{1} i_{2} \cdots i_{\ell}}$. More explicitly in the cases of $\ell=2,3$,

$$
\begin{aligned}
A_{<i j>} & =A_{(i j)}-\frac{1}{3} \delta_{i j} A_{k k} \\
A_{<i j k>} & =A_{(i j k)}-\frac{1}{5}\left(\delta_{i j} A_{(k m m)}+\delta_{j k} A_{(i m m)}+\delta_{k i} A_{(j m m)}\right) .
\end{aligned}
$$

The spherical harmonics expansion of a scalar function $A$ on the unit-sphere can be written as

$$
A=\sum_{\ell=0}^{\infty} A_{<i_{1} i_{2} \cdots i_{\ell}>} n^{<i_{1}} n^{i_{2}} \cdots n^{i_{\ell}>}
$$

where $n^{i}=X^{i} /|X|$. In this case, the order $\ell$, which is associated with the angular dependence, is equivalent to the total angular momentum, $J$. Thus the $J$ mode of the $(T T)$-component of the metric perturbation is totally determined by its angular dependence. Namely, the terms in the $(T T)$-component of the metric perturbation which contain

$$
\text { 1, } n^{i}, \quad n^{<i} n^{j>}
$$

correspond to the $J=0,1,2$ modes, respectively.

Next we consider the expansion of a vector field $A_{i}$,

$$
A_{i}=\sum_{\ell=0}^{\infty} A_{i<i_{1} i_{2} \cdots i_{\ell}>} n^{<i_{1}} n^{i_{2}} \cdots n^{i_{\ell}>}
$$

In this case the term of the $\ell$-th order in the angular dependence is decomposed into $J=\ell+1, \ell$ and $\ell-1$. This is done by using the Clebsch-Gordan reduction formula 14,

$$
U_{i} T_{i_{1} i_{2} \cdots i_{\ell}}=R_{i<i_{1} i_{2} \cdots i_{\ell}>}^{(+)}+\frac{\ell}{\ell+1} \epsilon_{j i<i_{\ell}} R_{i_{1} i_{2} \cdots i_{\ell-1}>j}^{(0)}+\frac{2 \ell-1}{2 \ell+1} \delta_{i<i_{\ell}} R_{i_{1} i_{2} \cdots i_{\ell-1}>}^{(-)}
$$

where $T_{i_{1} i_{2} \cdots i_{\ell}}$ is a STF tensor of order $\ell$ and

$$
\begin{aligned}
R_{i_{1} i_{2} \cdots i_{\ell+1}}^{(+)} & :=U_{<i_{\ell+1}} T_{i_{1} i_{2} \cdots i_{\ell}>} \\
R_{i_{1} i_{2} \cdots i_{\ell}}^{(0)} & :=U_{j} T_{k<i_{1} i_{2} \cdots i_{\ell-1}} \epsilon_{i_{\ell}>j k}, \\
R_{i_{1} i_{2} \cdots i_{\ell-1}}^{(-)} & :=U_{j} T_{j i_{1} i_{2} \cdots i_{\ell-1}} .
\end{aligned}
$$

We perform the decomposition explicitly for $\ell \leq 2$ here. For $\ell=0$, there exists no $J=0$ mode and it trivially corresponds to the $J=1$ mode. For $\ell=1$, the decomposition is performed as

$$
A_{i j} n^{j}=\left[\left(A_{(i j)}-\frac{1}{3} \delta_{i j} A_{k k}\right)+A_{[i j]}+\frac{1}{3} \delta_{i j} A_{k k}\right] n^{j},
$$

and the first, second and third terms in the square brackets correspond to the $J=2,1$ and 0 modes, respectively. For $\ell=2$, we obtain the decomposition formula as

$$
A_{i<j k>} n^{<j} n^{k>}=\left[A_{<i j k>}+\frac{2}{3} \epsilon_{m i<j} B_{k>m}^{(2)}+\frac{3}{5} \delta_{i<j} B_{k>}^{(1)}\right] n^{<j} n^{k>},
$$

where

$$
\begin{aligned}
B_{i j}^{(2)} & =\frac{1}{2}\left(A_{k<m i>} \epsilon_{j k m}+A_{k<m j>} \epsilon_{i k m}\right), \\
B_{k}^{(1)} & =A_{i<j k>} \delta_{i j}
\end{aligned}
$$


and the first, second and third terms correspond to the $J=3,2$ and 1 modes, respectively.

As an example, let us consider a vector,

$$
\frac{d z^{\alpha}}{d T} R_{\alpha \beta \gamma \delta} f^{\beta}{ }_{j}{ }_{i}{ }_{i} f_{k}{ }_{k} X^{j} X^{k},
$$

which appears in the $\left(\begin{array}{l}2 \\ 0\end{array}\right)$ and $\left(\begin{array}{l}2 \\ 1\end{array}\right)$ matchings in section 4 . First we decompose it in terms of its angular dependence as

$$
\frac{d z^{\alpha}}{d T} R_{\alpha \beta \gamma \delta} f_{i}^{\gamma}\left(f^{\beta}{ }_{j j} f_{k>}^{\delta} X^{<j} X^{k>}+\frac{1}{3} f_{k}^{\beta} f_{k}^{\delta}|X|^{2}\right) .
$$

Using the relation (4.16) and the fact that the Ricci tensor vanishes, the second term in the parentheses is rewritten as

$$
\frac{1}{3} \frac{d z^{\alpha}}{d T} R_{\alpha \beta \gamma \delta} f_{i}^{\gamma} \frac{d z^{\beta}}{d T} \frac{d z^{\delta}}{d T}|X|^{2},
$$

and is found to be zero due to the symmetry of the Riemann tensor. Thus we have only to consider the first term in the parenthesis of Eq. (C12), which is decomposed further with the aid of the formulas (C10) and (C9) as

$$
\frac{d z^{\alpha}}{d T} R_{\alpha \beta \gamma \delta}\left(f^{\gamma}{ }_{<i} f^{\beta}{ }_{j} f_{k>}^{\delta}+\frac{2}{3} \epsilon_{m i<j} F_{k>m}^{(2) \gamma \beta \delta}+\frac{3}{5} \delta_{i<j} F_{k>}^{(1) \gamma \beta \delta}\right) X^{<j} X^{k>},
$$

where

$$
\begin{aligned}
F_{i j}^{(2) \gamma \beta \delta} & :=\frac{1}{2}\left(f^{\gamma}{ }_{m} f^{\beta}{ }_{<n} f^{\delta}{ }_{i>} \epsilon_{j m n}+f^{\gamma}{ }_{m} f^{\beta}{ }_{<n} f^{\delta}{ }_{j>} \epsilon_{i m n}\right), \\
F_{i}^{(1) \gamma \beta \delta} & :=\frac{1}{2}\left(f^{\gamma}{ }_{k} f^{\beta}{ }_{i} f^{\delta}{ }_{k}+f^{\gamma}{ }_{k} f^{\beta}{ }_{k} f^{\delta}{ }_{i}\right)-\frac{1}{3} f^{\gamma}{ }_{i} f^{\beta}{ }_{k} f^{\delta}{ }_{k} .
\end{aligned}
$$

It is easy to see that the first and third terms vanish due to the symmetry of the Riemann tensor and the Ricci flatness. Thus only the $J=2$ mode remains.

As for a tensor field, it is not necessary for us to give general discussion here. The only term which requires our consideration is

$$
R_{\alpha \beta \gamma \delta} f_{i}^{\alpha} f_{k}^{\beta} f_{j}^{\gamma} f^{\delta}{ }_{m} X^{k} X^{m},
$$

which appears in the $(i j)$-component of the $\left(\begin{array}{l}2 \\ 0\end{array}\right)$ matching. In this case, it is better to use the symmetry of the Riemann tensor from the beginning. First, we define the spatial triad components of the Riemann tensor by

$$
R_{i j k m}:=R_{\alpha \beta \gamma \delta} f^{\alpha}{ }_{i} f_{j}^{\beta} f_{k} f^{\delta}{ }_{m} .
$$

Introducing a symmetric tensor defined by

$$
\mathcal{R}_{i j}=\frac{1}{4} \epsilon_{i k m} \epsilon_{j n s} R_{k m n s},
$$

we can express $R^{i k j m}$ in terms of $\mathcal{R}_{i j}$ as

$$
R_{i j k m}=\epsilon^{n i j} \epsilon^{s k m} \mathcal{R}_{n s} .
$$

Then the symmetric tensor $\mathcal{R}_{i j}$ is decomposed into STF tensors as

$$
\mathcal{R}_{i j}=\mathcal{R}_{<i j>}+\frac{1}{3} \delta_{i j} \mathcal{R}_{k k} .
$$

Counting the number of indices, we find that the first and second terms in Eq. ( $\overline{\mathrm{C} 20}$ ) correspond to $J=2$ and 0 modes, respectively. However, again owing to the symmetry of the Riemann tensor and the Ricci flatness, the $J=0$ mode vanishes and only the $J=2$ mode remains.

[1] K. S. Thorne, in Proceedings of the 8th Nishinomiya-Yukawa Meomorial Symposium: Relativistic Cosmology, ed. M. Sasaki (Universal Academy Press, Tokyo, 1994), p67, and the references therein. 
[2] K. Danzmann et al., 'LISA: Proposal for a Laser-Interferometric Gravitational Wave Detector in Space', MPQ-177 (1993), unpublished.

[3] C. Cutler et al., Phys. Rev. Lett. 70, 2984 (1994), and the references therein.

[4] H. Tagoshi, M. Shibata, T. Tanaka and M. Sasaki, Phys. Rev. D in press, Caltech preprint GRP-434/Osaka University preprint OU-TAP 28, gr-qc/9603028, and the references therein.

[5] B. S. DeWitt and R. W. Brehme, Ann. Phys. (N.Y.) 9, 220 (1960).

[6] P. D. D'Eath, Phys. Rev. D11, 1387 (1975).

[7] K. S. Thorne, and J. B. Hartle, Phys. Rev. D31, 1815 (1985).

[8] F. J. Zerilli, Phys. Rev. D2, 2141 (1970).

[9] C. M. DeWitt and B. S. DeWitt, Physics 1, 3 (1964).

[10] B. F. Shutz and C. M. Will, Astrophys. J. 291, L33 (1985).

[11] D. V. Gal'tsov, J. Phys. A15, 3737 (1982).

[12] A. Ori, Phys. Lett. A 202, 347 (1995).

[13] M. Carmeli, Phys. Rev. 138, B1003 (1965);

A. G. Smith and C. M. Will, Phys. Rev. D22, 1276 (1980).

[14] e.g., Appendix A of L. Blanchet and T. Damour, Philos. Trans. R. Soc. London A320, 379 (1986). 